\title{
Regular Apeirotopes of Dimension and Rank 4
}

\author{
Peter McMullen
}

Received: 24 April 2008 / Revised: 5 March 2009 / Accepted: 8 April 2009 /

Published online: 20 May 2009

(C) Springer Science+Business Media, LLC 2009

\begin{abstract}
In previous papers, all the four-dimensional (finite) regular polytopes have been classified, as well as the regular apeirotopes of full rank (that is, of rank 5). Of the two problems in $\mathbb{E}^{4}$ thus left open (namely, regular apeirotopes of ranks 3 and 4), this paper describes the regular apeirotopes of rank 4. The methods employed here are somewhat different from those in earlier work; while knowledge of the possible dimension vectors $\left(\operatorname{dim} R_{0}, \ldots, \operatorname{dim} R_{3}\right)$ of the mirrors $R_{0}, \ldots, R_{3}$ of the generating reflexions of the symmetry groups plays a rôle, the crystallographic restriction leads to a considerable emphasis being placed on the vertex-figures.
\end{abstract}

Keywords Polytope $\cdot$ Abstract $\cdot$ Regular $\cdot$ Realization · Apeirotope $\cdot$ Rank · Dimension

\section{Introduction}

This paper is part of a continuing programme, namely, to classify all the faithfully realized regular polytopes and discrete regular apeirotopes in each dimension. Stated so baldly, for many reasons this programme is far too ambitious. In small dimensions, however, the classification may be feasible: it has been settled in dimensions up to 3 , and here we deal with one of the two hitherto open cases in dimension 4 .

The context of the paper is that of realized regular polytopes, the general background to whose theory is described in [20, Chap. 5]. There are two quite different ways to approach realizations. The first, for which a fairly complete theory exists (at least, in the finite case), asks for a description of the space of all realizations of a given abstract regular polytope or apeirotope, with rank playing only a minor rôle (see [20, Sects. 5B, 5C] for further details). The second, about which much less is known in general terms, asks for a classification of the realizations of all these polytopes and

P. McMullen $(\varangle)$

University College London, Gower Street, WC1E 6BT London, England, UK

e-mail: p.mcmullen@ucl.ac.uk 
apeirotopes in a euclidean space of given dimension (in this case, it is usual to impose conditions such as faithfulness and discreteness).

The finite regular polyhedra have long been known; adding to the Petrie-Coxeter apeirohedra of [3], Grünbaum [8] found all but one of the remaining regular apeirohedra, while Dress $[5,6]$ found the missing example and proved that the classification was then complete. In [19] (see also [20, Sects. 7E, 7F]), the present author and Schulte classified completely all the faithfully realized regular polytopes and discrete regular apeirotopes in dimensions up to three, including a quick proof of Dress's result. Further, in [13], this author classified the regular polytopes and apeirotopes of full (that is, maximal) rank in each higher dimension. Finally, in [14], this author found all the four-dimensional (finite) regular polyhedra (the polyhedra with planar faces were-in effect-classified in [1,2]).

In four dimensions, the currently open problems are those of classifying the regular apeirohedra and 4-apeirotopes. The present paper settles the second of these problems, partly using similar techniques to those of the earlier papers. The core of the previous argument involved determining the possible sequences $\left(\operatorname{dim} R_{0}, \ldots, \operatorname{dim} R_{3}\right)$ - the dimension vectors - of the mirrors of the reflexions $R_{0}, \ldots, R_{3}$ that generate the symmetry groups of the regular apeirotopes. However, the crystallographic criterion implies that there are relatively few possible symmetry groups of the vertex-figures, and so while we bear the dimension vectors in mind, it is ultimately on the latter that we concentrate.

Let us briefly outline the rest of the paper. In Sect. 2 we describe those parts of the general theory of regular polytopes and their realizations that we need. In [14] we introduced a general notation for regular polygons; in Sect. 3 we extend this to regular apeirogons. Section 4 is devoted to various operations that lead from one regular polytope or apeirotope to another. Certain of the apeirotopes are blended, and so arise from three-dimensional ones; we treat these in Sect. 5. In Sect. 6 we discuss the possible vertex-figures of our apeirotopes; in particular, we show that a pure regular apeirotope of dimension and rank 4 cannot have a three-dimensional vertexfigure. However, the case of blended vertex-figures is very interesting; the concept of cluster polytopes leads to fairly explicit geometric descriptions of the corresponding apeirotopes. The remaining three sections deal with the actual classification of the apeirotopes, split up according to their groups.

It is worth observing that our analysis has yielded many new examples of faithfully realized universal regular polyhedra and apeirotopes, in the sense explicated in Sect. 2.

\section{Regular Polytopes}

For the general background on abstract regular polytopes, we refer the reader to the monograph [20]; for the most part, we shall not cite original papers on the theory of abstract regular polytopes directly. In this paper, we largely concentrate on the geometric aspects of the theory, that is, on realized regular polytopes.

There are many candidates for spaces in which regular polytopes $\mathcal{P}$ might be realized geometrically. The usual (and generally most useful) context of realizations is 
of those in euclidean spaces, because it is in these that we obtain the richest structure. (Note, however, that some basic results have a common statement if they are posed for realizations in spherical or hyperbolic spaces as well; see [13, Sect. 3].)

It is a familiar fact (see [20, Theorem 2E11]) that an abstract regular polytope $\mathcal{P}$ of rank $n$ (or $n$-polytope for short) can be identified with its automorphism group, which is a string $C$-group $\Gamma=\left\langle\rho_{0}, \ldots, \rho_{n-1}\right\rangle$ generated by $n$ involutions $\rho_{j}$ (the distinguished generators) such that $\rho_{j}$ and $\rho_{k}$ commute if $0 \leqslant j \leqslant k-2 \leqslant n-3$, and

$$
\left\langle\rho_{i} \mid i \in \mathrm{J}\right\rangle \cap\left\langle\rho_{i} \mid i \in \mathrm{K}\right\rangle=\left\langle\rho_{i} \mid i \in \mathrm{J} \cap \mathrm{K}\right\rangle
$$

for any $\mathrm{J}, \mathrm{K} \subseteq \mathrm{N}:=\{0, \ldots, n-1\}$; the last is the intersection property. The $j$-faces of $\mathcal{P}$ are then the right cosets $\Gamma_{j} \sigma$ of the distinguished subgroup

$$
\Gamma_{j}:=\left\langle\rho_{i} \mid i \neq j\right\rangle
$$

for each $j \in \mathrm{N}$, and two faces are incident just when they intersect (as cosets). In fact, incidence actually induces an order relation:

$$
\Gamma_{j} \sigma \leqslant \Gamma_{k} \tau \Longleftrightarrow \Gamma_{j} \sigma \cap \Gamma_{k} \tau \neq \emptyset \quad \text { and } \quad j \leqslant k .
$$

Formally, we also adjoin two copies of $\Gamma$ itself, labelled $\Gamma_{-1}$ and $\Gamma_{n}$, as the (unique) $(-1)$ - and $n$-faces of $\mathcal{P}$.

The maximal chains (with respect to this ordering) are the flags of an abstract polytope $\mathcal{P}$; the group $\Gamma$ is then simply transitive on the flags of $\mathcal{P}$. (Note that we do not usually mention the $(-1)$ - and $n$-faces in flags.) Call two flags $j$-adjacent if they differ by their $j$-face. With appropriate connectedness conditions related to adjacency on the poset of faces of $\mathcal{P}$ (see [20, Sect. 2A]; we do not reproduce the technical details), the transitivity condition defines the regularity of $\mathcal{P}$. For $j=0, \ldots, n-1$, associated with the base flag $\Phi:=\left\{\Gamma_{0}, \ldots, \Gamma_{n-1}\right\}$ is the distinguished generator $\rho_{j}$, which takes $\Phi$ into the $j$-adjacent flag $\Phi^{j}$.

Note that the distinguished subgroups $\Gamma_{n-1}=\left\langle\rho_{0}, \ldots, \rho_{n-2}\right\rangle$ and $\Gamma_{0}=\left\langle\rho_{1}, \ldots\right.$, $\left.\rho_{n-1}\right\rangle$ are themselves string C-groups; the corresponding polytopes are the facet and vertex-figure of $\mathcal{P}$, respectively.

To avoid cases which, in our context, turn out to be trivial, we usually assume that adjacent generators $\rho_{j-1}$ and $\rho_{j}$ of $\Gamma$ do not commute (this is justified almost immediately). In other words, the period $p_{j}$ of the product $\rho_{j-1} \rho_{j}$ satisfies $p_{j}>2$; in theory, $p_{j}=\infty$ for any $j$ is allowable, but here-because vertex-figures have to be finite-this can only happen for $j=1$. We call $\left\{p_{1}, \ldots, p_{n-1}\right\}$ the Schläfli type of the polytope. If the polytope is determined just by the $p_{j}$, then we have the universal regular polytope (of that Schläfli type), for which we use the same symbol $\left\{p_{1}, \ldots, p_{n-1}\right\}$ (but without qualification); we write $\left[p_{1}, \ldots, p_{n-1}\right]$ for the corresponding Coxeter group. Generally, however, the group $\Gamma$ will satisfy additional relations as well, for some of which we introduce special notation later.

In the geometric context (that is, of realizations, for the general background to which, see $[9,11,16]$ or $\left[20\right.$, Chap. 5]), each $\rho_{j}$ is represented (under some homomorphism into the group $\mathcal{I}$ of isometries of a euclidean space) by a reflexion $R_{j}$-an involutory isometry - which we identify with its mirror of fixed points

$$
\left\{x \mid x R_{j}=x\right\} .
$$


We write $\mathcal{G}:=\left\langle R_{0}, \ldots, R_{n-1}\right\rangle$ for the corresponding symmetry group. The intersection property (2.1) translates directly into

$$
\left\langle R_{i} \mid i \in \mathrm{J}\right\rangle \cap\left\langle R_{i} \mid i \in \mathrm{K}\right\rangle=\left\langle R_{i} \mid i \in \mathrm{J} \cap \mathrm{K}\right\rangle .
$$

The structure of the realization $P$ is then given by Wythoff's construction, namely, the initial vertex is $F_{0}:=v \in R_{1} \cap \cdots \cap R_{n-1}$ (the latter is called the Wythoff space), and, recursively, the initial $j$-face is

$$
F_{j}:=\left\{F_{j-1} G \mid G \in\left\langle R_{0}, \ldots, R_{j-1}\right\rangle\right\}
$$

for $j=1, \ldots, n-1$; all other (proper) faces are images of the initial faces under $\mathcal{G}$. Further, $\Gamma_{-1}$ and $\Gamma_{n}$ are represented by $F_{-1}:=\emptyset$ and $F_{n}:=P$. The partial ordering of the faces is induced by iterated membership: thus, $F_{-1} \in F_{0} \in \cdots \in F_{d}=P$. The (affine) subspace

$$
E:=\operatorname{aff}\{v G \mid G \in \mathcal{G}\}
$$

spanned by the vertices of $P$ is called the ambient space of $P$; we lose no generality in thinking of this as the whole space in which we work. We then write $\operatorname{dim} P:=\operatorname{dim} E$, which is the dimension of $P$. Finally, the realization is faithful if $P$ (as a poset) is isomorphic to $\mathcal{P}$; it cannot be faithful if any $p_{j}=2$ (see [9] or [20, Theorem 5A7]). In practice, since we always work with the group $\mathcal{G}$ of isometries rather than the abstract group $\Gamma$, faithfulness will actually mean that geometric degeneracies do not occur and that the mapping induced by $\rho_{j} \mapsto R_{j}$ is injective. We also refer to a faithful realization of some abstract regular polytope as polytopal; note that realizations need not be faithful, or even polytopal.

A realization of an abstract regular polytope $\mathcal{P}$ determines a realization of each of its faces or vertex-figures. In particular, $F_{n-1}$ (and its induced structure, with the same initial vertex $v$ ) gives a realization of the facet of $\mathcal{P}$; its symmetry group is the image $\mathcal{G}_{n-1}$ of $\Gamma_{n-1}$. If we write $w$ for the mid-point of the edge between $v$ and $v R_{0}$, then $w$ is the initial vertex of a realization of the vertex-figure of $\mathcal{P}$, with symmetry group the image $\mathcal{G}_{0}=\left\langle R_{1}, \ldots, R_{n-1}\right\rangle$ of $\Gamma_{0}$. Faithfulness is hereditary; that is, if the original realization of $\mathcal{P}$ is faithful, then the realizations of the facet and vertex-figure of $\mathcal{P}$ are also faithful.

We sometimes find it more convenient to use $v R_{0}$ rather than $w$ as the initial vertex of the vertex-figure $Q$, which just replaces $Q$ by its scaled-up copy $2 Q-v$ relative to $v$. For most purposes, this makes little difference, since the combinatorics are not altered, and we have the added convenience that the vertices of the vertexfigure occur among those of the polytope itself. In the present case, we are dealing with apeirotopes (that is, infinite polytopes); it is then usual to take the initial vertex $v=F_{0}$ to be the origin $o$ of coordinates, so that the group $\mathcal{G}_{0}$ of the vertex-figure is an orthogonal group.

There is no harm in adopting the intuitive view-point that an edge is the (line-)segment joining its two vertices and that a 2-face is the polygon determined by its constituent (vertices and) edges. Fortunately, our approach will not need us to visualize faces of higher rank. It is, though, helpful to note that infinite facets of regular apeirotopes of full rank 5 in $\mathbb{E}^{4}$ must occur in the list that we are about to 
compile; in particular, the three Petrie-Coxeter apeirohedra of [3] will themselves be facets of such apeirotopes.

There are important restrictions on faithful realizations, which we state in general terms; we refer to [20, Sects. 5B, 5C] for proofs.

Theorem 2.1 Let $P$ be a faithful realization of an abstract regular apeirotope $\mathcal{P}$ whose ambient space $E$ is euclidean. Then $\operatorname{dim} P \geqslant \operatorname{rank} \mathcal{P}-1$.

Theorem 2.2 Let $P$ be a faithful realization of an abstract regular $n$-apeirotope in a euclidean space $E$ with group $\mathcal{G}=\left\langle R_{0}, \ldots, R_{n-1}\right\rangle$. Then $\operatorname{dim} R_{j} \geqslant j$ for $j=$ $0, \ldots, n-2$, and $\operatorname{dim} R_{n-1} \geqslant n-2$.

The sequence $\left(\operatorname{dim} R_{0}, \operatorname{dim} R_{1}, \ldots, \operatorname{dim} R_{n-1}\right)$ is called the dimension vector of the realization. A first step in the classification of the faithfully realized regular polytopes of a fixed rank $n$ in a fixed dimension $d$ is to determine which dimension vectors can occur. We have already solved this problem in [14] for the finite case with $\left(R_{0}, R_{1}, R_{2}\right)$ in $E=\mathbb{E}^{4}$; we shall merely quote those results where appropriate and not repeat the analysis.

If we have (not necessarily faithful or even polytopal) realizations of the abstract regular polytope (or apeirotope) $\mathcal{P}$ in two euclidean spaces, say $P$ with mirrors $S_{0}, \ldots, S_{n-1}$ in $L$ and $Q$ with mirrors $T_{0}, \ldots, T_{n-1}$ in $M$ (possibly some $S_{j}=L$ or $T_{j}=M$ ), then their blend has mirrors $S_{j} \times T_{j}$ in $L \times M$ for $j=0, \ldots, n-1$. Indeed, if $v \in S_{1} \cap \cdots \cap S_{n-1}$ and $w \in T_{1} \cap \cdots \cap T_{n-1}$ are the initial vertices of the two realizations, then $(v, w)$ can be chosen as the initial vertex of the blend, which we then write $P$ \# $Q$. A realization that cannot be expressed as a blend in a non-trivial way is called pure.

There is a last restriction on realizations of apeirotopes which we wish to impose, that of discreteness. (In general, a discrete realization that is a blend can have nondiscrete components, but this will not happen here.) This means that $P$ has a discrete vertex-set $v \mathcal{G}$, and hence a finite vertex-figure; thus $\mathcal{G}$ itself is crystallographic. The special group of $P$ is the subgroup $\mathcal{S}:=\left\langle S_{0}, \ldots, S_{n-1}\right\rangle$ of the orthogonal group, where (identified with its mirror) $S_{j}:=R_{j}-R_{j}$ for each $j$; of course, $S_{j}$ is the translate of $R_{j}$ that contains $o$. (With the convention $F_{0}=o$, we have $S_{j}=R_{j}$ for $j \geqslant 1$.) Then $\mathcal{S}$ must be crystallographic and, in particular, must be finite and cannot have ordinary rotations (with an axis of codimension 2) of periods other than 2, 3, 4, or 6.

We end the section with a general remark. If $S$ and $T$ are linear reflexions, then, since $S T=(-S)(-T)=S^{\perp} T^{\perp}$ (thus identifying $-S$ with its mirror $S^{\perp}$, and so on), $S \cap T$ and $S^{\perp} \cap T^{\perp}$ are both pointwise fixed by the product. That is, the axis (fixed set) of $S T$ is

$$
(S \cap T)+\left(S^{\perp} \cap T^{\perp}\right)=(S \cap T)+(S+T)^{\perp} .
$$

In particular, if $S$ and $T$ commute, then (2.2) is the mirror of their product $S T=T S$, which is again a reflexion. There is an obvious generalization to any two reflexions whose mirrors meet. 


\section{Polygons and Apeirogons}

In [14] we introduced notation to describe the type of a regular polygon. Here, we recall this notation and extend it to certain regular apeirogons. Using the notation, the geometric structure of regular polyhedra and apeirohedra can also be described, at least in a rough and ready fashion, and this then allows a similar description of regular polytopes and apeirotopes of higher ranks.

As is well known (see, for example, [20, p. 135]), a regular $n$-gon $P$ (in some euclidean space) is expressible in a unique way as a blend of planar $n$-gons. Some of these may degenerate into $m$-gons, with $m$ a divisor of $n$; the case $m=2$ - the line segment $\{2\}$ is allowed as a particular case. (For reasons that will soon become clear, in this paper we shall write $\{2\}$ rather than-as in [20] and earlier papers -\{\} for the segment, genuinely thinking of it as a degenerate polygon.) The basic rotational symmetry of $P$-permuting the vertices of $P$ cyclically-is then a composition of rotations through angles $2 \pi d_{j} / n$ for some $1 \leqslant d_{1}<\cdots<d_{k} \leqslant n / 2$, with $d_{k}=n / 2$ indicating $\{2\}$; we assume that the greatest common divisor $\left(d_{1}, \ldots, d_{k}, n\right)=1$. The notation for $P$ (which suppresses the relative sizes of the component polygons) is then

$$
P=\left\{\frac{n}{d_{1}, \ldots, d_{k}}\right\}:=\left\{\frac{n}{d_{1}}\right\} \# \ldots \#\left\{\frac{n}{d_{k}}\right\},
$$

although, in writing $P$ as a blend, the individual fractions $n / d_{j}$ will always be reduced. With the understanding that $p$ may stand for a generalized fraction of the above kind, namely,

$$
p=\frac{n}{d_{1}, \ldots, d_{k}}
$$

we then write $P=\{p\}$.

We can easily extend this notation to apeirogons, at least in the cases that arise here (we do not have components with rotations through irrational multiples of $\pi$ ). Such an apeirogon is a blend of one of the above kind with the (linear) apeirogon $\{\infty\}$. We then use the same notation as before, but we now allow $0 \leqslant d_{1}<\cdots<d_{k} \leqslant n / 2$ to encompass the case $d_{1}=0$ indicating an apeirogonal component. Thus, for example, $\left\{\frac{2}{0,1}\right\}$ is a zigzag apeirogon, while $\left\{\frac{3}{0,1}\right\}$ is a helical apeirogon that winds around an infinite triangular cylinder. (The unblended linear apeirogon $\{\infty\}$ cannot occur as a face, but it would be $\left\{\frac{1}{0}\right\}$ in this notation.)

The notation extends to polyhedra (or apeirohedra-we do not yet distinguish the cases): $\{p, q\}$ denotes a polyhedron whose faces are polygons of type $\{p\}$ and vertexfigures of type $\{q\}$. (Of course, we could then extend it even further to polytopes of higher rank, but we shall introduce more precise descriptions of polyhedra in Sect. 4, leading to more precise descriptions of our apeirotopes.)

It is useful to introduce a little more notation here, in anticipation of Sect. 4. As we have seen, a regular polygon or apeirogon is of the form

$$
\{p\}=\left\{p_{1}\right\} \# \cdots \#\left\{p_{k}\right\}
$$


for some (usually fractional) $\infty \geqslant p_{1}>\cdots>p_{k} \geqslant 2$ (this ordering respects the previous ordering $0 \leqslant d_{1}<\cdots<d_{k} \leqslant n / 2$ of the denominators of the fractional expression for $p$ ). If $2 \leqslant q \leqslant \infty$, define $q^{\prime \prime}$ by

$$
\frac{1}{q}+\frac{1}{q^{\prime \prime}}=\frac{1}{2},
$$

where $2^{\prime \prime}=\infty\left(\right.$ and $\left.\infty^{\prime \prime}=2\right)$; we then set

$$
\left\{p^{\prime \prime}\right\}:=\left\{p_{k}^{\prime \prime}\right\} \# \cdots \#\left\{p_{1}^{\prime \prime}\right\}
$$

with the appropriate reversal of ordering. Of course, $\left(p^{\prime \prime}\right)^{\prime \prime}=p$. Observe that, if $n, n^{\prime \prime}$ are the numerators of $p, p^{\prime \prime}$ as generalized fractions, then we can have $n^{\prime \prime}=2 n, n$, or $n / 2$. We say that the polygons (or apeirogons) $\{p\}$ and $\left\{p^{\prime \prime}\right\}$ are complementary or are complements; recall that complementary angles add up to $\pi / 2$ and that the angle between the mirrors of the reflexions that generate the group of $\{q\}$ is $\pi / q$.

\section{Operations}

In this section, we describe various operations on the generating reflexions that lead (at least potentially) from one regular apeirotope to another. Most of these are on the groups of the vertex-figure, although they naturally affect the whole apeirotope. One, however, while preserving the initial edge, alters the reflexion $R_{0}$ that interchanges its two vertices. We also do some general calculations on types of faces, and so on, which will save us having to do them later in individual cases.

We begin by working in greater generality than we subsequently need. Let $P$ be a regular $n$-polytope (or apeirotope) with symmetry group $\mathcal{G}=\mathcal{G}(P)=$ $\left\langle R_{0}, \ldots, R_{n-1}\right\rangle$, initial vertex $v \in R_{1} \cap \cdots \cap R_{n-1}$, and initial edge $E:=\left\{v, v R_{0}\right\}$. With $P$ are associated various regular polygons, whose vertices and edges are among those of $P$. Thus, to specify such a polygon, we need to describe its generating reflexions $S_{0}$ and $S_{1}$; we shall set things up in such a way that the initial vertex and edge are $v$ and $E$. There are two basic polygons.

- Petrie polygon. For this,

$$
S_{0}:=R_{0} R_{2} R_{4} \cdots, \quad S_{1}:=R_{1} R_{3} R_{5} \cdots .
$$

There is a recursive picture of a Petrie polygon: each $n-1$ successive edges, but no $n$, are edges of a Petrie polygon of a facet of $P$; the Petrie polygon of a polygon is that polygon itself (because, as the definition shows, $S_{0}=R_{0}$ and $S_{1}=R_{1}$ ). If an abstract regular polytope of Schläfli type $\left\{p_{1}, \ldots, p_{n-1}\right\}$ is determined solely by the length $r$ of its Petrie polygon, then we denote it by $\left\{p_{1}, \ldots, p_{n-1}\right\}_{r}$.

- Deep hole. For this,

$$
S_{0}:=R_{0}, \quad S_{1}:=R_{1} R_{2} R_{3} \cdots R_{n-2} R_{n-1} R_{n-2} \cdots R_{3} R_{2} R_{1} .
$$

There is no neat description of a deep hole analogous to that of a Petrie polygon. If an abstract regular polytope of Schläfli type $\left\{p_{1}, \ldots, p_{n-1}\right\}$ is determined solely by the length $h$ of its deep hole, then we denote it by $\left\{p_{1}, \ldots, p_{n-1} \mid h\right\}$. 
Remark 4.1 Petrie polygons have long been known and used. Deep holes were introduced in [12]; see also [20, Sect. 7D].

When $n=3$ (the case of polyhedra), there are generalizations of Petrie polygons and holes. Regarding a regular polyhedron as the vertex-figure of a 4-apeirotope $P$, we denote it by $Q$ and take it to be of Schläfli type $\{q, r\}$ with group $\mathcal{G}_{0}=\mathcal{G}(Q)=$ $\left\langle R_{1}, R_{2}, R_{3}\right\rangle$. (Similar considerations apply to the facet $F$ of $P$ with group $\mathcal{G}_{3}=$ $\mathcal{G}(F)=\left\langle R_{0}, R_{1}, R_{2}\right\rangle$, except that its Schläfli type $\{p, q\}$ can have $p=\infty$.)

- The group of the $k$-zigzag is given by

$$
S_{1}:=R_{1} R_{3}, \quad S_{2}:=\left(R_{2} R_{3}\right)^{k-1} R_{2},
$$

so that the 1-zigzag is the Petrie polygon.

- The group of the $k$-hole is given by

$$
S_{1}:=R_{1}, \quad S_{2}:=\left(R_{2} R_{3}\right)^{k-1} R_{2},
$$

so that the 1-hole is just the face. Since the 2-hole (that is, the deep hole when $n=3$ ) is of most interest to us, we usually refer to it as just the hole.

If a regular polyhedron $Q$ has zigzags and holes of specified kinds, then we write

$$
Q=\left\{q, r: s_{1}, s_{2}, \ldots \mid t_{2}, t_{3}, \ldots\right\} .
$$

Here, the $k$-zigzag is of type $\left\{s_{k}\right\}$, and the $k$-hole is of type $\left\{t_{k}\right\}$. There are various conventions governing this usage:

- a redundant entry is replaced by '.'

- a string $s_{1}, \ldots, s_{m}$ or $t_{2}, \ldots, t_{n}$ terminates at the last non-redundant entry

- if no zigzags (or holes) are specified, then the corresponding delimiter ':' (or '|') is omitted

The general notation is asymmetric between zigzags and holes.

Remark 4.2 If $k^{\prime} \equiv \pm k(\bmod r)$, then $k$ - and $k^{\prime}$-zigzags or holes are the same. Further, if $r=2 k$ is even, then $k$-zigzags and $k$-holes are geometrically the same, although the initial generators $S_{1}$ are different.

Remark 4.3 It may happen that $Q$ is actually determined (up to isomorphism) by the specification of the lengths of certain zigzags and holes. If so, it will be the universal regular polyhedron of this type and is then described by listing just the numerators of the fractions in its notation. For example, as an abstract polytope, the great dodecahedron $\left\{5, \frac{5}{2}\right\}$ is actually $\{5,5 \mid 3\}$. As above, we denote a universal polyhedron determined by its hole or Petrie polygon in the traditional way, without fractional entries, and with the Petrie polygon indicated by a suffix (as $\{6,3\}_{4}$ for the Petrial of the cube $\{4,3\})$.

Remark 4.4 In earlier work, we would have written $Q=\left\{q, r \mid t_{2}, \ldots, t_{n}\right\}_{s_{1}, \ldots, s_{m}}$. We have varied the notation for two reasons: first, to make it more symmetric between 
holes and zigzags and, second, because we frequently have (generalized) fractional entries $s_{j}$ that are not very legible as suffixes.

Remark 4.5 The notation $\{P, Q\}$ for a polytope (or apeirotope) whose facet is of type $P$ and whose vertex-figure is of type $Q$ (with the vertex-figure of $P$ isomorphic to the facet of $Q$ ) is well established. It is occasionally appropriate to indicate the type $\{s\}$ of the Petrie polygon or $\{t\}$ of the deep hole as well; in an extension of the previous notation, we write $\{P, Q: s \mid t\}$, with the same convention as before if we specify only one of the two.

We now move on to operations on polytopes (or apeirotopes). In general terms, a (mixing) operation $\rho$ on a regular $n$-polytope $P$ with group $\mathcal{G}=\mathcal{G}(P)$ as before constructs a new regular polytope $P^{\rho}$, whose group $\mathcal{G}\left(P^{\rho}\right)=\mathcal{G}^{\rho}$ is generated by certain reflexions in $\mathcal{G}$. Often, but not necessarily, $P^{\rho}$ will be of the same rank $n$; this will happen with many of our operations because they are involutory. The corresponding operation on the generators is written

$$
\rho:\left(R_{0}, \ldots, R_{n-1}\right) \mapsto\left(S_{0}, \ldots, S_{m-1}\right),
$$

where the $S_{j}$ are the generators of the new group. (We shall occasionally write $R_{j}^{\rho}$ instead of $S_{j}$ to avoid the need to repeat generators that have not changed.) Although we do not usually think of it as such, strictly speaking duality, given by

$$
\delta:\left(R_{0}, \ldots, R_{n-1}\right) \mapsto\left(R_{n-1}, \ldots, R_{0}\right)
$$

is also an operation.

Remark 4.6 Many of our apeirotopes do not have (regular) duals in the geometric sense, although (of course) they always have abstract duals. In such a case, we shall simply say that the apeirotope has no dual, or that it is not dualizable.

From now on, we mostly confine our attention to the cases $n=3$ and 4 , starting with the former (as we have said, usually regarded as vertex-figures). The (involutory) Petrie operation is defined by

$$
\pi:\left(R_{1}, R_{2}, R_{3}\right) \mapsto\left(R_{1} R_{3}, R_{2}, R_{3}\right) .
$$

We call $Q^{\pi}$ the Petrial of $Q$. (It may happen that $Q^{\pi}$ is not a genuine polyhedron when $Q$ is; one example is the torus $Q=\{3,6\}_{(1,1)}$ with 3 vertices of [20, p. 19]. However, such cases are themselves almost degenerate, and we shall not meet any of them here.)

Remark 4.7 Observe that the Petrie operation $\pi$ interchanges $k$-zigzags and $k$-holes. In other words, if $Q$ has certain specified zigzags and holes as in (4.1), then

$$
Q^{\pi}=\left\{s_{1}, r: q, t_{2}, t_{3}, \ldots \mid s_{2}, s_{3}, \ldots\right\} .
$$


Next, suppose that $Q$ has centre $o$. For $j=1,2,3$, the operation $\kappa_{j}$ replaces $R_{j}$ by its orthogonal complement $R_{j}^{\perp}$, while keeping unchanged the generators $R_{k}$ with $k \neq j$. In terms of the group, this is equivalent to replacing $R_{j}$ by $-R_{j}=(-I) \cdot R_{j}$, where $-I$ is the reflexion (inversion) in $\{o\}$.

Remark 4.8 For $j=2,3$, there are no general rules relating the faces and vertexfigures of $Q$ and $Q^{\kappa_{j}}$; observe that the period of a product involving $R_{j}$ can stay the same, halve, or double. Note also that $\kappa_{j}$ is not permitted if $\operatorname{dim} R_{j}^{\perp}$ falls foul of the restrictions of Theorem 2.2; when $j=2,3$, this confines the operation to the case $\operatorname{dim} R_{j}=2$. We return to $\kappa_{1}$ below.

The operation of halving applies if $q=4$; it is

$$
\eta:\left(R_{1}, R_{2}, R_{3}\right) \mapsto\left(R_{1} R_{2} R_{1}, R_{3}, R_{2}\right) .
$$

There are cases where the new polyhedron $Q^{\eta}$ degenerates (we shall note these in the appropriate places). If it exists, then $Q^{\eta}$ is self-dual; the duality is induced by conjugating the generators by $R_{1}$. Geometrically, the vertex-sets satisfy vert $Q^{\eta} \subseteq$ vert $Q$; moreover, the initial vertex of $Q^{\eta}$ is the same as that of $Q$. If all the edgecircuits of $Q$ are even, then $Q^{\eta}$ has exactly half as many vertices as $Q$, and the order of the group $\mathcal{G}_{0}$ is halved; if $Q$ has odd edge-circuits, then vert $Q^{\eta}=$ vert $Q$, and the group remains the same. The edges of $Q^{\eta}$ are diagonals of the square faces of $Q$, and the faces are its vertex-figures. As an example, if $C$ is the cube $\{4,3\}$ and $T$ is the tetrahedron $\{3,3\}$, then $C^{\eta}=T$. Note further that the rôles of $R_{2}$ and $R_{3}$ in the vertex-figure are reversed, the Petrie polygon of $Q^{\eta}$ corresponds to the 2-zigzag of the dual of $Q$; moreover, if the Petrie polygon of $Q$ is of type $\{h\}$, then the hole of $Q^{\eta}$ is of type $\left\{\frac{1}{2} h\right\}$ (that with alternate vertices of $\{h\}$ ).

We have another operation that applies to the vertex-figure $Q$ if $q=4$; this is

$$
\varsigma:\left(R_{1}, R_{2}, R_{3}\right) \mapsto\left(R_{2}, R_{1} R_{3},\left(R_{1} R_{2}\right)^{2}\right) .
$$

This is actually the operation $\sigma$ of [19], called skewing or skew halving, but applied to the dual: $\varsigma=\delta \sigma$ (this explains why we have used the variant form of sigma to denote it). We have changed the definition of the operation in this way, because (as reference to [14] shows) $\varsigma$ works on actual polyhedra, whereas $\sigma$ often only operates on groups. (When $Q$ is a torus, and so self-dual, it does not matter whether we apply $\sigma$ or $\varsigma$, and in one place we actually prefer the former.) There is no immediate geometric relationship between $Q$ and $Q^{\varsigma}$; its Schläfli type $\left\{q^{\prime}, r^{\prime}\right\}$ is given by the lengths $q^{\prime}$ of the Petrie polygon of $Q$ and $r^{\prime}$ of the 2-hole of $Q^{\delta}$ (the dual of $Q$ ). We discuss the individual cases in the appropriate places.

We now suppose that $P$ is a 4 -apeirotope in $\mathbb{E}^{4}$ and look at possible operations on $P$. The Petrie operation on the vertex-figure $Q$ of $P$ induces one on $P$ itself, denoted by the same symbol. Thus the operation is

$$
\pi:\left(R_{0}, \ldots, R_{3}\right) \mapsto\left(R_{0}, R_{1} R_{3}, R_{2}, R_{3}\right) .
$$

From the definition we obtain the following. 
Theorem 4.9 If $P$ is of Schläfli type $\{p, q, r\}$, then $\pi$ cannot lead to a new apeirotope $P^{\pi}$ when $p$ is odd. When $\pi$ is valid, then

- the vertices, edges, and faces of $P^{\pi}$ are the same as those of $P$,

- the holes of facets of $P^{\pi}$ are the same as those of $P$,

- the Petrie polygons offacets of $P^{\pi}$ are the same as Petrie polygons of $P$ itself (and vice versa).

Proof For the first claim, if $p$ is odd, then

$$
\left(R_{0} \cdot R_{1} R_{3}\right)^{p}=R_{3} \quad \Longrightarrow \quad R_{3} \in\left\langle R_{0}, R_{1} R_{3}\right\rangle,
$$

contrary to the intersection property. The remaining claims are straightforward to prove; note that $R_{3}$ commutes with $R_{0}$ and $R_{1}$.

We now suppose that $o$ is the centre of the vertex-figure $Q$ of our 4-apeirotope $P$ in $\mathbb{E}^{4}$ (and so usually the initial vertex of $P$ itself). For $j \geqslant 1$, we extend the operation $\kappa_{j}$ in the obvious way, so that $\kappa_{j}$ replaces $R_{j}$ by $-R_{j}=R_{j}^{\perp}$ (as a mirror). We observed in Remark 4.8 that, if $j=2,3$, then $\kappa_{j}$ can only apply if $\operatorname{dim} R_{j}=2$. Since its occurrence is the most frequent, we usually write $\kappa$ instead of $\kappa_{1}$.

In fact, there is a far-reaching generalization of $\kappa$, which we shall find very useful. Let $P$ be any regular polytope (finite or infinite), whose group is generated by the reflexions $R_{0}, R_{1}, \ldots$ Let $K$ denote the point-reflexion in the initial vertex of $P$, denote by $\kappa$ the operation

$$
\kappa:\left(R_{0}, R_{1}, R_{2}, \ldots\right) \mapsto\left(R_{0}, R_{1} K, R_{2}, \ldots\right),
$$

and write $P^{\kappa}$ for the corresponding regular polytope (if it exists, of course). Various properties of $\kappa$ follow easily from the definition.

Theorem 4.10 Under the operation $\kappa$ :

(a) $(P \# Q)^{\kappa}=P^{\kappa} \# Q^{\kappa}$ for any regular polytopes $P$ and $Q$;

(b) $\{p\}^{\kappa}=\left\{p^{\prime \prime}\right\}$ for $2 \leqslant p \leqslant \infty$;

(c) if $j \geqslant 2$ and $F$ is the (initial) $j$-face of $P$, then $F^{\kappa}$ is the (initial) $j$-face of $P^{\kappa}$.

Recall that $p^{\prime \prime}$ is defined by $1 / p+1 / p^{\prime \prime}=1 / 2$.

Proof Part (a) is straightforward: if $P$ has initial vertex $v$ and $Q$ has initial vertex $w$, then the product of the point-reflexions in $v$ and $w$ is the point-reflexion in $(v, w)$. For (b), consider first the extreme cases $p=2$ or $\infty$. For $\{2\}$, we must define $R_{1}=E$, the ambient line of the segment; the group of $\{2\}^{\kappa}$ is then $\left\langle R_{0}, K\right\rangle$, namely, the group of $\{\infty\}$. Since $\kappa$ is involutory (or direct), we see that $\{\infty\}^{\kappa}=\{2\}$. If $2<p<\infty$, take the initial vertex of $\{p\}$ to be $o$ and compare the effects of $R_{0} R_{1}$ and $R_{0}\left(R_{1} K\right)$. If $v=o R_{0}$ is taken by $R_{1}$ into $w=v R_{1}$, then $v$ is taken by $R_{1} K$ into $v\left(-R_{1}\right)=-w$; it follows that the angles of $\{p\}$ and $\{p\}^{\kappa}$ at $o$ are supplementary. Since the angle of $\{p\}$ at its vertex is $(1-2 / p) \pi$, it follows immediately that $\{p\}^{\kappa}=\left\{p^{\prime \prime}\right\}$, as required. Part (c) is again straightforward; the condition $j \geqslant 2$ merely ensures that there actually is a reflexion $R_{1}$. 
Remark 4.11 We shall see examples of applying $\kappa$ later. For the moment, we merely note that (as applied to facets) $\kappa$ interchanges ordinary regular polyhedra and the Petrie-Coxeter apeirohedra, and their Petrials and other three-dimensional regular apeirohedra:

$$
\begin{gathered}
\{3,3\} \stackrel{\kappa}{\longleftrightarrow}\left\{6, \frac{6}{1,3} \mid 3\right\} \cong\{6,6 \mid 3\}, \\
\{3,4\} \stackrel{\kappa}{\longleftrightarrow}\left\{6, \frac{4}{1,2} \mid 4\right\} \cong\{6,4 \mid 4\}, \\
\{4,3\} \stackrel{\kappa}{\longleftrightarrow}\left\{4, \frac{6}{1,3} \mid 4\right\} \cong\{4,6 \mid 4\}, \\
\left\{\frac{4}{1,2}, 3: 3\right\}=\{3,3\}^{\pi} \stackrel{\kappa}{\longleftrightarrow}\left\{\frac{3}{0,1}, \frac{6}{1,3}: 4,4\right\} \cong\{\infty, 6\}_{4,4}, \\
\left\{\frac{6}{1,3}, 4: 3\right\}=\{3,4\}^{\pi} \stackrel{\kappa}{\longleftrightarrow}\left\{\frac{3}{0,1}, \frac{4}{1,2}: 6,4\right\} \cong\{\infty, 4\}_{6,4}, \\
\left\{\frac{6}{1,3}, 3: 3\right\}=\{4,3\}^{\pi} \stackrel{\kappa}{\longleftrightarrow}\left\{\frac{4}{0,1}, \frac{6}{1,3}: 6,3\right\} \cong\{\infty, 6\}_{6,3} .
\end{gathered}
$$

The notation is that of [19]. Further, since $\pi$ and $\kappa$ commute here as well, we have

$$
\left\{6, \frac{6}{1,3} \mid 3\right\}^{\pi}=\left\{\frac{3}{0,1}, \frac{6}{1,3}: 4,4\right\},
$$

and so on.

We deduce the effect of $\kappa$ on an apeirotope from Theorem 4.10, where $\left\{p^{\prime \prime}\right\}$ was defined in (3.1) when $p$ is a generalized fraction.

Theorem 4.12 Let $\left\{s_{k}\right\}$ and $\left\{t_{k}\right\}$ be the types of the $k$-zigzag and $k$-hole of the facet of $P$, respectively. Then the types of the $k$-zigzag and $k$-hole of the facet of $P^{\kappa}$ are

$$
\begin{aligned}
& \left\{s_{k}\right\},\left\{t_{k}\right\}, \quad \text { respectively, } \quad \text { if } k \text { is even, } \\
& \left\{s_{k}^{\prime \prime}\right\},\left\{t_{k}^{\prime \prime}\right\}, \quad \text { respectively, if } k \text { is odd. }
\end{aligned}
$$

Proof For this, we just observe that, because $K$ commutes with $R_{2}$, under $\kappa$ we have

$$
\left(R_{1} R_{2}\right)^{k-1} R_{1} \mapsto\left(R_{1} K R_{2}\right)^{k-1} R_{1} K=\left(R_{1} R_{2}\right)^{k-1} R_{1} \cdot K^{k} ;
$$

we then appeal to the definitions of the $k$-zigzag and $k$-hole, and all three parts of Theorem 4.10.

Remark 4.13 Since $\kappa$ can double or halve the number of vertices of the vertex-figure, there are occasions when its application will result in a degenerate apeirotope. Indeed, we shall take advantage of this in Sect. 6 and its applications.

Remark 4.14 It is clear that $\pi$ and $\kappa$ commute (when both are applicable): $\pi \kappa=\kappa \pi$.

We similarly write $P^{\eta}$ for the effect on $P$ of applying the halving operation to its vertex-figure. We next describe some features of $P^{\eta}$, which follow directly from the definition. 
Theorem 4.15 Let $P$ be a regular 4-apeirotope of Schläfli type $\{p, 4, r\}$. If $P^{\eta}$ exists, then

- vertices and edges of $P^{\eta}$ are among those of $P$,

- faces of $P^{\eta}$ are holes of facets of $P$,

- holes of the facets of $P^{\eta}$ are deep holes of $P$,

- if, in addition, $p=3$, then facets of $P^{\eta}$ are vertex-figures of $P$.

Proof All the claims except for the last follow easily. For the last assertion, conjugating the generators of the facet of $P^{\eta}$ by $R_{1} R_{0}$ (which moves the initial vertex of $P$ to that of the vertex-figure of $P$ ) and using the fact that $R_{0} R_{1} R_{0} R_{1} R_{0}=R_{1}$, with $\mathcal{G}\left(P^{\eta}\right)$ generated by $S_{0}, \ldots, S_{3}$ we obtain

$$
R_{0} R_{1}\left(S_{0}, S_{1}, S_{2}\right) R_{1} R_{0}=R_{0} R_{1}\left(R_{0}, R_{1} R_{2} R_{1}, R_{3}\right) R_{1} R_{0}=\left(R_{1}, R_{2}, R_{3}\right),
$$

since $R_{1}$ commutes with $R_{3}$ and $R_{0}$ commutes with $R_{2}$ and $R_{3}$.

Remark 4.16 Since $\left(-R_{1}\right) R_{2}\left(-R_{1}\right)=R_{1} R_{2} R_{1}$, it is clear that $\kappa \eta=\eta$. However, it may be possible to apply $\kappa$ after $\eta$.

As we have observed, there is no direct geometric connexion between $Q$ and $Q^{S}$ (thought of as putative vertex-figures), and it thus makes little sense to write $P \mapsto P^{\varsigma}$ for a corresponding action on apeirotopes.

A final operation changes $R_{0}$. If $P$ has Schläfli type $\{p, q, r\}$ with $r$ even, define $T:=\left(R_{2} R_{3}\right)^{r / 2}$, and the operation $\tau$ by

$$
\tau:\left(R_{0}, \ldots, R_{3}\right) \mapsto\left(R_{0} T, R_{1}, R_{2}, R_{3}\right) .
$$

In a sense, this corresponds to the operation denoted by $\kappa_{02}$ in [13], although that was defined in geometric terms. Once again, there are no general formulae relating $P$ and $P^{\tau}$.

Remark 4.17 It may happen that $\tau$ is defined for one apeirotope, but not for a closely related one (for example, the vertices of the second vertex-figure may be a subset of those of the first). The same reflexion $T$ will then exist, and so we shall use the same symbol $\tau$ for the replacement of $R_{0}$ by $R_{0} T$. Of course, there is no reason to suppose that, because $\tau$ yields a new apeirotope for old in one case, then it will necessarily do so in another.

Remark 4.18 In our subsequent analysis, it is sometimes the case that all that we can say about a new apeirotope $P^{\rho}$ derived by an operation $\rho$ from another one $P$ is a consequence of one of the descriptive theorems in this section. In such a case, when all we know about the new facet is the types of its face and vertex-figure, and perhaps some zigzags or holes, we shall merely note its existence. The cases that we do comment on will then be of greater interest. For example, the new facet may be a universal polyhedron or apeirohedron, or closely related to one, or the whole apeirotope could be universal. 


\section{The Blended Cases}

The blended cases are easily dealt with. In our case, the only possible dimensions for the components are 3 and 1, since two components of dimensions 2 cannot provide us with a blend of rank 4 . The one-dimensional component is a segment $\{2\}$ or an apeirogon $\{\infty\}$, and it follows that the three-dimensional component has to be a discrete regular 4-apeirotope.

Remark 5.1 The last statement needs a little justification, because a priori it is not obvious that the three-dimensional component has to be polytopal. However, it must satisfy the criterion of [13] for the dimension vector (only polytopality of the vertexfigure is assumed). Moreover, the only thing that can force degeneracy is to have an odd edge-circuit and a non-centrally symmetric vertex-figure; this is impossible, since the latter can only be the tetrahedron or its Petrial, and those vertex-figures only permit the "apeir" construction of the next Sect. 6. Compare Remark 8.7, where the contrary situation prevails.

These 4-apeirotopes were classified in [19]; see also [13], where they are placed in the context of regular polytopes of full rank. They are of two kinds according as $\operatorname{dim} S_{0}=2$ or 0 (in [13], it was shown that these are the only possibilities), and further fall into pairs related by the Petrie operation on their vertex-figures (giving $\operatorname{dim} S_{1}=2$ or 1 , again the only possibilities-recall from Sect. 2 the convention that the generating reflexions of the components are $S_{j} \leqslant \mathbb{E}^{3}$ and $T_{j} \leqslant \mathbb{R}$ ). Note that $\operatorname{dim} S_{2}=\operatorname{dim} S_{3}=2$ only. When $\operatorname{dim} S_{0}=0$, they are the result of applying the apeir construction (which we describe in the next Sect. 6) to a suitable rational polyhedron, and in this case blending with $\{2\}$ yields an isomorphic apeirotope (the vertices lie in parallel hyperplanes, and edges go from one hyperplane to the other). However, this isomorphism only results because no three-dimensional regular 4-apeirotope has odd edge-circuits.

The dimension vectors of $\{2\}$ and $\{\infty\}$ are $\left(\operatorname{dim} T_{0}, \ldots, \operatorname{dim} T_{3}\right)=(0,1,1,1)$ and $(0,0,1,1)$, respectively; remember that we have to take $\operatorname{dim} T_{j}=\operatorname{dim} E$-where $E=\mathbb{R}$ is the ambient space-for those $j$ beyond the range of the actual generating reflexions. Thus the dimension vectors of the blends are the various sums of four possibilities for the $\operatorname{dim} S_{j}$ and two for the $\operatorname{dim} T_{j}$; hence we obtain $8+8=16$ examples of blends in all, which there is little point in listing.

\section{The Vertex-Figures}

As we have said, we shall not repeat the analysis of [14]; in particular, we refer to that paper for some of the concepts that we use subsequently. Instead of following the pattern of [14], we look for the crystallographic groups and then ensure that each corresponding vertex-figure is subsequently considered.

An important general method derives a regular apeirotope from a suitable finite regular polytope. This is the free abelian apeirotope or "apeir" construction, which we have described in $[13,14]$; we give a brief description here. 
Initially, the construction applies to any finite regular polytope $Q$. Let $Q$ sit in the euclidean space $E$ - not necessarily its ambient space-and let its symmetry group be $\left\langle R_{1}, R_{2}, \ldots\right\rangle$ (we begin the labelling at 1 deliberately). Let $w$ be the initial vertex of $Q$; define $R_{0}:=\{w\}$ (recall that this stands for the point-reflexion as well) and pick any $v \in R_{1} \cap R_{2} \cap \cdots$. Then there is a regular apeirotope $P:=$ apeir $Q$ with $\left(R_{0}, R_{1}, R_{2}, \ldots\right)$ as the generating reflexions of its symmetry group $\mathcal{G}(P), v$ as initial vertex, and $Q$ as vertex-figure.

However, $P$ will not generally be discrete. This condition holds only if $Q$ is $r a$ tional, meaning that the vertices in its vertex-set $W=$ vert $Q$ all have rational coordinates with respect to some basis of $E$. To see this, just note that the vertex-set of $P$ is

$$
\text { vert } P=\{v, 2 w-v\}+\left\{2 n\left(w^{\prime}-w\right) \mid w^{\prime} \in W \text { and } n \in \mathbb{Z}\right\} .
$$

In turn, this forces $\mathcal{G}(Q)$ to consist of rational matrices with respect to the same basis of $E$ (taking $v=o$ ), so that $Q$ is crystallographic.

It may seem that there could be a great deal of choice for the initial vertex $v$. In fact, this is largely illusory. In reality, what matters is whether $v \in$ aff $W$ or not. In the former case, $\operatorname{dim} P=\operatorname{dim} Q$, and in the latter, $\operatorname{dim} P=\operatorname{dim} Q+1$. And, indeed, the latter case can be obtained from the former by blending with a segment; for this, we refer to the previous Sects. 2 and 5.

In view of this last remark, therefore, we need only consider the apeirotopes apeir $Q$ with $Q$ a full-dimensional regular polyhedron. In what follows, we shall take for granted the fact that each vertex-figure $Q$ admits an apeirotope apeir $Q$.

Remark 6.1 Observe that, if $Q$ is a polyhedron of type $\{q, r\}$ with $r$ even, then there is an applicable operation $\tau$, and hence (at least in principle) an apeirotope $P$ with vertex-figure $Q$ and initial reflexion $R_{0}$ with $\operatorname{dim} R_{0}>0$.

We now establish an important restriction. The three-dimensional analogues of our apeirotopes are the regular apeirohedra. As has already been mentioned, the easiest method of classifying them was described in [19], where the concept of dimension vector (of the mirrors of the generating reflexions) was first introduced. However, there are other ways of looking at the classification. One that did not play an important rôle there was to distinguish between those whose vertex-figures are threedimensional and those that are planar. In the present context, it is natural to ask specifically whether it is possible to have a three-dimensional vertex-figure when the apeirotope is not blended (of course, there are certainly blended examples, as we have seen). Our next task is to answer this question in the negative.

So, suppose that $P$ is a regular 4-apeirotope whose vertex-figure $Q$ is threedimensional. As usual, the group of $P$ is $\mathcal{G}(P)=\left\langle R_{0}, \ldots, R_{3}\right\rangle$ with the $R_{j}$ the canonical generating reflexions; we continue to take $o$ as the initial vertex. Let $H:=\operatorname{aff} Q(=\operatorname{aff}$ vert $Q)$, so that $H$ is a hyperplane, and let $v$ be the initial vertex of $Q$. Let $S$ be the hyperplane through $v$ with normal $v$; since reflexion in $R_{0}$ interchanges $o$ and $2 v$, it is clear that $v \in R_{0} \subseteq S$.

In fact, we can say more. Let $T:=S \cap H$, and let $L \subset S$ be the line in $S$ through $v$ perpendicular to $T$. Since no (affine) subspace of $T$ except $\{v\}$ is fixed by both 
$R_{2}$ and $R_{3}$ (because they do not commute), it follows that $R_{0} \cap H=\{v\}$ or $T$. We conclude that $R_{0}=\{v\}, L, T$, or $S$, according as $\operatorname{dim} R_{0}=0,1,2$, or 3 .

The case $R_{0}=\{v\}$ yields $P=$ apeir $Q$ (whether or not $o \in H$ ), which is threedimensional or a blend. The case $R_{0}=T$ similarly yields either a three-dimensional apeirotope (if $o \in H$ ) or a blend (otherwise). For the remaining two cases, the apeirotope is three-dimensional if $o \in H$; otherwise, $R_{0}$ meets the line $M$ through $o$ perpendicular to $H$, yielding a fixed point of the whole group, in which case $P$ is finite.

We summarize this discussion in

Theorem 6.2 Apart from blends, there are no four-dimensional regular apeirotopes of rank 4 whose vertex-figures are three-dimensional.

A slight modification of the foregoing argument deals with the case of a blended vertex-figure $Q$. In this case, there are (distinct) parallel hyperplanes $\pm H$ that contain vert $Q$; with $v$ the initial vertex of $Q$, we can suppose as before that $v \in H$. Once again, we have the same four possibilities for $R_{0}$. With the previous notation, the case $\operatorname{dim} R_{0}=0$ (so that $R_{0}=\{v\}$ ) gives $P=$ apeir $Q$. If $\operatorname{dim} R_{0}=2$ (so that $R_{0}=T$ ), then we easily see that $P$ is a blend; recalling Sect. 5, we conclude that the only possibilities are $P=\{4,3,4\} \#\{\infty\}$ or its image under the Petrie operation $P^{\pi}=\left\{\left\{4, \frac{6}{1,3} \mid 4\right\},\left\{\frac{6}{1,3}, 4: 3\right\}\right\} \#\{\infty\}$ (recall from [19] that $\left\{\left\{4, \frac{6}{1,3} \mid 4\right\},\left\{\frac{6}{1,3}, 4: 3\right\}\right\} \cong$ $\left\{\{4,6 \mid 4\},\{6,4\}_{3}\right\}$ is universal).

We are thus left with the cases $\operatorname{dim} R_{0}=1$ (with $R_{0}=L$ ) or $\operatorname{dim} R_{0}=3$ (with $R_{0}=S$ ); the resulting group is then (affinely) irreducible. It is straightforward to see that, if $\operatorname{dim} R_{0}=3$, then (modulo Petriality of the vertex-figure) we have a group generated by hyperplane reflexions on which $R_{1}$ acts as an involutory automorphism. These hyperplane reflexions are $R_{0}, R_{2}, R_{3}$ and their conjugates $R_{1} R_{0} R_{1}$ and $R_{1} R_{2} R_{1}$, and lead to a diagram $\mathcal{A}_{1}(p, q, r, s)$, as shown in Fig. 1 . A small number $j$ indicates the reflexion $R_{j}$.

However, there are many similarities between the two cases, and so we give an alternative description, which helps to visualize what happens. First, we look more closely at the vertex-figure $Q$ and its group $\mathcal{G}_{0}=\mathcal{G}(Q)$. Up to Petriality, we can take $Q=\{q, r\} \#\{2\}$ for some Coxeter group $[q, r]$. For $Q=\{3, r\} \#\{2\}$, we have $\mathcal{G}_{0}=[3, r] \times \mathcal{C}_{2}$, where $\mathcal{C}_{2}=\left\langle H_{0}\right\rangle$, where $H_{0}$ (standing, as usual, for its mirror) is the hyperplane through $o$ (the initial vertex) parallel to $\pm H$. On the other hand, $\{4,3\} \#\{2\}$ has group $[3,3] \times \mathcal{C}_{2}$, where now $\mathcal{C}_{2}$ is generated by the reflexion $K:=-I$ in $o$. If we define the $R_{1}$-even subgroup $\mathcal{G}_{0}^{\mathrm{e}} \leqslant \mathcal{G}_{0}$ to be that whose elements expressed in terms of the generators have $R_{1}$ appearing an even number of times, then $\mathcal{G}_{0}^{\mathrm{e}}=[3, r]$ for some $r$; it is important to note that $[4,3]$ (in the dual form) does not occur.

Fig. $1 \mathcal{A}_{1}(p, q, r, s)$

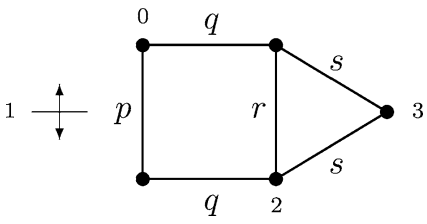


If $\mathcal{G}_{0}^{\mathrm{e}}=\left\langle S_{1}, S_{2}, S_{3}\right\rangle$ (say), then $S_{j}=R_{j}$ for $j=2,3$; for $Q=\{3, r\} \#\{2\}$, we have $R_{1}=S_{1} H_{0}$, but for $Q=\{4,3\} \#\{2\}$, we have to take $R_{1}=-S_{1} S_{3}=K S_{1} S_{3}$. Reversing the implicit operations, we can recover the $R_{1}$-even subgroup from

$$
S_{1}= \begin{cases}R_{2} R_{1} R_{2} R_{1} R_{2}, & \text { in case }\{3, r\} \#\{2\} \text { for } r=3,4, \\ R_{2} R_{1} R_{3} R_{2} R_{3} R_{1} R_{2}, & \text { in case }\{4,3\} \#\{2\} .\end{cases}
$$

Remark 6.3 We write these blends just as $\{3, r\} \#\{2\}=\left\{\frac{6}{2,3}, r\right\}$ and $\{4,3\} \#\{2\}=$ $\left\{\frac{4}{1,2}, 3\right\}$. For the last, we have $\left\{\frac{4}{1,2}, 3\right\} \cong\{4,3\}$. For $\left\{\frac{6}{2,3}, r\right\}$, the easiest way to specify its group $\left\langle R_{1}, R_{2}, R_{3}\right\rangle$ is as the quotient of $[6, r]$ by the relation requiring that $\left(R_{1} R_{2}\right)^{3}$ commute with $R_{3}$. As it happens, $\left\{\frac{6}{2,3}, 3\right\} \cong\{6,3\}_{(2,0)}$ (a torus) in the notation of [20, p. 19], while $\left\{\frac{6}{2,3}, 4\right\}$ is obtained by deleting the opposite squares of a truncated octahedron and identifying them by translation.

Now define $S_{0}:=R_{0}$ and consider the group $\left\langle S_{0}, S_{1}, S_{2}, S_{3}\right\rangle$; this is a finite group, because $S_{0}$ meets the symmetry axis $M$ of $Q$, in fact, in a single point $Z=S_{0} \cap M$. Thus, with initial vertex $o$, it gives rise to a regular 4-polytope $C$, which we call the cluster polytope. The group of $C$ is $\mathcal{G}(C)=\left\langle S_{0}, S_{1}, S_{2}, S_{3}\right\rangle$, where $C=\{p, q, r\}$ (if $\operatorname{dim} S_{0}=3$ ) or $\{p, q, r\}^{\zeta}$ (if $\operatorname{dim} S_{0}=1$ ) for some $p, q, r$; here, $\zeta$ replaces $S_{0}$ by $S_{0} Z$, with the centre $Z$ of $C$ regarded as a point-mirror (this is, in fact, the operation $\kappa_{0}$ of [13, Sect. 3], but we use a different notation to avoid any confusion with the $\kappa_{j}$ for $\left.j=1,2,3\right)$. Since $C$ is crystallographic and we do not have $q=4$, the only possibilities for $(p, q, r)$ are $(3,3,3),(3,3,4)$, or $(4,3,3)$. The polytope $C$ itself bears little relation to the original apeirotope $P$; however, its edge-set $E$ (which is a subset of the edge-set of $P$ itself), similarly called the edge-cluster, plays a crucial rôle in the geometry of $P$. Two different edge-clusters, which are images of the initial edge-cluster $E$ under $\mathcal{G}(P)$, meet in at most a single vertex and are then adjacent (thus $E$ meets the adjacent edge-cluster $E R_{0}$ in the initial vertex $o$ ); observe, in particular, that a face of $P$ goes successively from one edge-cluster to an adjacent one. We discuss the specific examples (and their geometry) in subsequent sections.

Remark 6.4 Informally, we can think of the edge-cluster $E$ as given by the initial vertex and that half of the vertex-figure $Q$ lying in the hyperplane $H$. There are two possible half vertex-figures related by Petriality, which yield the same edge-set.

We can work out various data about $P$ from those of $C$, although the occurrence of the hyperplane reflexion $H_{0}$ makes some calculations difficult. In fact, it is easier if we employ a further trick. In case $Q=\{4,3\} \#\{2\}$, we can write $S_{1}=-R_{1} R_{3}$. We therefore define $S_{1}^{\prime}:=-R_{1} R_{3}$ in the other two cases as well; thus $S_{1}^{\prime}$ is a hyperplane reflexion. For $Q=\{3,4\} \#\{2\}$, we actually have the same group [3, 4$] \times \mathcal{C}_{2}$, now with $\mathcal{C}_{2}$ generated by $K=-I$ (note that $K=\left(R_{1} R_{2} R_{3}\right)^{3}$ here). In case $Q=\{3,3\} \#\{2\}$, however, we obtain $[4,3] \times \mathcal{C}_{2}$. It might at first seem that passing to a larger group could cause problems; in fact, it does not, because (as we shall see in Sect. 8) $C=\{3,3,3\}$ is incompatible with $Q=\{3,3\} \#\{2\}$. We therefore have a false cluster polytope $C^{\prime}$ with group $\mathcal{G}\left(C^{\prime}\right)=\left\langle S_{0}, S_{1}^{\prime}, S_{2}, S_{3}\right\rangle$; in effect (even though $\kappa$ is, strictly 
speaking, an invalid operation), we have $C^{\prime}=P^{\kappa \pi}$. Again, we shall discuss the geometrical relationships in the appropriate places.

Reversing the definition yields $R_{1}=K S_{1}^{\prime} S_{3}$, which we can interpret (as in Sect. 4) as $P=\left(C^{\prime}\right)^{\kappa \pi}$. In fact, since we shall want $P^{\pi}=\left(C^{\prime}\right)^{\kappa}$ as well, to do the calculations it is easier to go through this intermediate stage. Whether $C^{\prime}$ is one of the classical regular 4-polytopes $\{p, q, r\}=\{3,3,3\},\{3,3,4\},\{4,3,3\}$, or $\{3,4,3\}$, or of the form $\{p, q, r\}^{\zeta}$, the vertex-figure of $\left(C^{\prime}\right)^{\kappa}$ is $\{q, r\} \diamond\{2\}$ in the notation of [14, Sect. 6]. To obtain their types, we appeal to the appropriate part of the table of that section:

$$
\begin{aligned}
& \{3,3\} \diamond\{2\}=\left\{\frac{6}{1,3}, 3: 4\right\} \#\{2\}=\left\{\frac{6}{1,3}, 3: \frac{4}{1,2}\right\}, \\
& \{3,4\} \diamond\{2\}=\left\{\frac{6}{1,3}, 4: 3\right\} \#\{2\}=\left\{\frac{6}{1,3}, 4: \frac{6}{2,3}\right\}, \\
& \{4,3\} \diamond\{2\}=\left\{\frac{4}{1,2}, 3: 3\right\} \#\{2\}=\left\{\frac{4}{1,2}, 3: \frac{6}{2,3}\right\} ;
\end{aligned}
$$

the last is just a cube $\{4,3\}$, but we specify the Petrie polygon $\left\{\frac{6}{2,3}\right\}$ to emphasise that it is geometrically distinct (note that it is not the blend of the ordinary cube with a segment). However, we also need to apply $\kappa$ to the facet of $\{p, q, r\}^{\zeta}$, which is also of the form $Q \diamond\{2\}$ for some three-dimensional polyhedron $Q$.

It is now straightforward to deduce relationships between $P$ and $C^{\prime}$.

Theorem 6.5 Let $P$ be a regular 4-apeirotope with blended vertex-figure, and let $C^{\prime}$ be the corresponding false cluster polytope.

- The face of $P$ is the complement of the face of $C^{\prime}$.

- The holes of facets and deep holes of $P$ and $C^{\prime}$ are the same.

- The Petrie polygon of the facet of $P$ is the complement of the Petrie polygon of $C^{\prime}$.

- The Petrie polygon of $P$ is the complement of the Petrie polygon of the facet of $C^{\prime}$.

Proof This result is just a combination of Theorems 4.12 and 4.9 in turn, and so we can read off the data from them.

Remark 6.6 Since the faces of $\left(C^{\prime}\right)^{\kappa}$ are either even-sided polygons or apeirogons, $\pi$ is always applicable, as we should expect.

Remark 6.7 The operation $\zeta$ is induced by reflexion in the centre of $C$, namely, the point $Z=S_{0} \cap S_{1} \cap S_{2} \cap S_{3}$. This changes $R_{0}$ into $R_{0} Z$ and is unaffected by the replacement of $C$ by the false cluster polytope $C^{\prime}$. We shall therefore use $\zeta$ to indicate the same change on $P$ and $P^{\pi}$.

Remark 6.8 Applying the operation $\eta$ to a 4-apeirotope $P$ with a blended vertexfigure would also result in a three-dimensional vertex-figure, thus yielding a blended apeirotope (if $\operatorname{dim} R_{0}=0$ or 2 ) or a finite polytope (if $\operatorname{dim} R_{0}=1$ or 3 ). 
Remark 6.9 It may help keep track of the various operations if we bear in mind the corresponding dimension vectors:

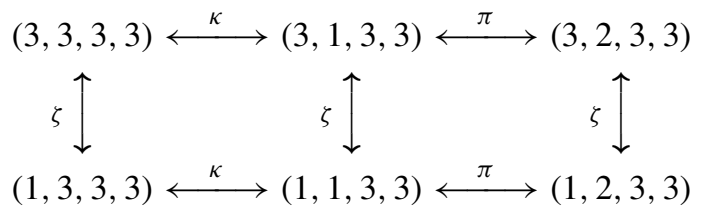

The starting point at top left is a classical regular 4-polytope, while that at top right is obtained from a diagram $\mathcal{A}_{1}$; moreover, the facet at top middle is a Petrie-Coxeter apeirohedron (in two cases, that at top right is as well).

Remark 6.10 Strictly speaking, there are two further (finite) crystallographic regular 4-polytopes, namely, $P:=\{4,3,3\}^{\pi}=\left\{\left\{4, \frac{4}{1,2} \mid 4\right\},\left\{\frac{4}{1,2}, 3: 3\right\}\right\} \cong\left\{\{4,4 \mid 4\},\{4,3\}_{3}\right\}$ and $P^{\zeta}$. However, since $\pi \kappa=\kappa \pi$, we obtain nothing new.

Since we can henceforth assume that the crystallographic group $\mathcal{G}(P)$ is irreducible, it must be a subgroup of one of the following: first, $\left(\mathcal{V}_{3} \times \mathcal{V}_{3}\right) \rtimes \mathcal{C}_{2}$ with $\mathcal{V}_{3}:=[3,6]$ the group of the tiling of $\mathbb{E}^{2}$ by equilateral triangles and $\mathcal{C}_{2}$ the cyclic group of order 2 acting as an involutory automorphism (switching the two components $\mathcal{V}_{3}$ ); second, $\mathcal{P}_{5} \rtimes \mathcal{D}_{5}$ with

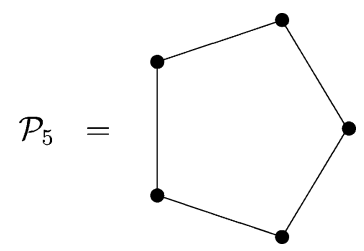

the (infinite) Coxeter group with diagram an unmarked pentagon and $\mathcal{D}_{5}$ the dihedral group of order 10 acting on it by the obvious diagram automorphisms (in practice, only $\mathcal{P}_{5} \rtimes \mathcal{C}_{2}$ occurs); third, $\mathcal{U}_{5}:=[3,3,4,3]$, the Coxeter group



which is the symmetry group of the tiling of $\mathbb{E}^{4}$ by 4 -cross-polytopes or 24-cells. (We shall subsume groups such as $[4,3,3,4],[4,3,3,4] \rtimes \mathcal{C}_{2}$, and $([4,4] \times[4,4]) \rtimes \mathcal{C}_{2}$ under the last.)

Therefore, the group of a potential vertex-figure $Q$ must be a group $[p, q] \times \mathcal{C}_{2}$, where $[p, q]=[3,3]$ or $[3,4]$, and $\mathcal{C}_{2}$ is the cyclic group of order 2 or a subgroup of one of $\left(\mathcal{D}_{q} \times \mathcal{D}_{q}\right) \rtimes \mathcal{C}_{2}$ for $q=3,4,6$, where $\mathcal{D}_{q}$ is the dihedral group of or$\operatorname{der} 2 q,[3,3,3] \rtimes \mathcal{C}_{2}=[3,3,3] \times \mathcal{C}_{2}$ with $[3,3,3]$ the symmetry group of the 4simplex (here, $\mathcal{C}_{2}$ is generated by $-I$ ), or $[3,4,3]$, the group of the regular 24-cell. Note that the extended group $[3,4,3] \rtimes \mathcal{C}_{2}$ is not crystallographic; of course, the group $[3,3,5]$ of the regular 600 -cell is not crystallographic either. In practice, the cases $[p, q] \times \mathcal{C}_{2}$ and $\left(\mathcal{D}_{4} \times \mathcal{D}_{4}\right) \rtimes \mathcal{C}_{2}$ can (with one exception) be subsumed under 
$[3,4,3]$; further, the cases $\left(\mathcal{D}_{q} \times \mathcal{D}_{q}\right) \rtimes \mathcal{C}_{2}$ with $q=3,6$ are naturally to be considered together.

\section{The Case $\left(\mathcal{V}_{3} \times \mathcal{V}_{3}\right) \times \mathcal{C}_{2}$}

For the vertex-figure, the starting point in this case is the torus

$$
\left\{4, \frac{4}{1,2} \mid 6\right\} \cong\{4,4 \mid 6\},
$$

whose vertices are those of the (orthogonal direct) product $\{6\} \times\{6\}$ of two congruent regular hexagons. It is convenient here to use complex coordinates $z=(x, y)$ with $x, y \in \mathbb{C}$ further regarded as pairs of real numbers. Writing $\omega:=\exp (2 i \pi / 3)=$ $(-1+i \sqrt{3}) / 2$, so that $\varphi:=-\omega^{2}=\exp (i \pi / 3)$ is a sixth root of one, the vertex-set of $\left\{4, \frac{4}{1,2} \mid 6\right\}$ is $D_{1}:=\left\{\left(\varphi^{m}, \varphi^{n}\right) \mid 0 \leqslant m, n<6\right\}$. Its group $\mathcal{G}_{0}$ then has generators

$$
\begin{aligned}
& R_{1}: z \mapsto(\varphi \bar{x}, y), \\
& R_{2}: z \mapsto(y, x), \\
& R_{3}: z \mapsto(x, \bar{y}) .
\end{aligned}
$$

The initial vertex is $(1,1)$, and the initial edge joins $(1,1)$ to $(\varphi, 1)$.

Remark 7.1 It is worth recalling that the isomorphism class of a regular torus of (Schläfli) type $\{4,4\}$ is determined by the number of its vertices. If this is $r^{2}$, then the torus is $\{4,4 \mid r\}$; if it is $2 r^{2}$, then the torus is $\{4,4\}_{2 r}$.

Remark 7.2 While we shall consider the torus $\left\{4, \frac{4}{1,2} \mid 4\right\}$ and most of its relatives in Sect. 9, it will turn out to be convenient to treat $\left\{4, \frac{4}{1,2} \mid 4\right\}^{5}$ in this section.

Successive applications of halving $\eta$ lead to

$$
\left\{\frac{4}{1,2}, 4: \frac{6}{1,3}\right\}, \quad\left\{4, \frac{4}{1,2} \mid 3\right\}, \quad\left\{\frac{4}{1,2}, 4: \frac{6}{2,3}\right\} ;
$$

the first has vertex-set $D_{2}:=\left\{\left(\varphi^{m}, \varphi^{n}\right) \mid 0 \leqslant m, n<6\right.$ and $m+n$ even $\}$, while the last two have the same vertex-set $D_{3}:=\left\{\left(\omega^{m}, \omega^{n}\right) \mid 0 \leqslant m, n<3\right\}$ (and hence the same group-indeed, $\left\{\frac{4}{1,2}, 4: \frac{6}{2,3}\right\}^{\eta}=\left\{4, \frac{4}{1,2} \mid 3\right\}$ again). We retain the initial vertex $(1,1)$, which is now joined by the initial edge to $(\varphi, \varphi),(\omega, 1)$, or $(\omega, \omega)$, respectively (after passing to suitable conjugate sets of generators).

Remark 7.3 As a torus, $\left\{\frac{4}{1,2}, 4: \frac{6}{2,3}\right\} \cong\{4,4 \mid 3\}$. However, a careful check shows that the corresponding hole symmetry has period 6 rather than 3 , so that the hole is, strictly speaking, a doubly-covered triangle.

Certainly, we have the free abelian apeirotope apeir $Q$ based on each of these polyhedra $Q$. But, at least for the initial polyhedron, we have a non-trivial symmetry 
Fig. 2 The diagram $\mathcal{A}_{2}(p, r)$

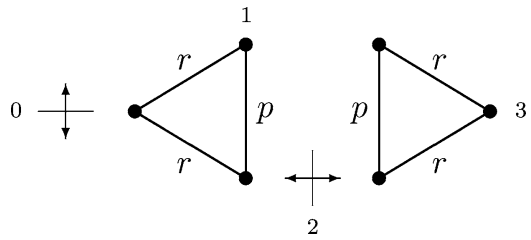

$T=\left(R_{2} R_{3}\right)^{2}: z \mapsto(\bar{x}, \bar{y})$ with $\operatorname{dim} T=2$; applying the corresponding operation $\tau$ to apeir $Q$, we have a new reflexion

$$
R_{0}: z \mapsto(1-\bar{x}, 1-\bar{y}) .
$$

With this $R_{0}$ and the appropriate $R_{1}, R_{2}, R_{3}$ coming from any of the other vertexfigures, we always obtain a discrete group, but not necessarily a non-degenerate apeirotope.

With the initial reflexions, we actually have a twisted Coxeter group with diagram $\mathcal{A}_{2}\left(\frac{3}{2}, 6\right)$, where the general diagram $\mathcal{A}_{2}(p, r)$ is as in Fig. 2. Thus $R_{1}$ and $R_{3}$ (whose dimensions are 3 ) correspond to the marked nodes of the diagram, while $R_{0}$ and $R_{2}$ (whose dimensions are 2) correspond to the indicated diagram automorphisms. The resulting apeirotope is $\left\{\left\{\frac{6}{2,3}, 4: \frac{4}{1,2}\right\},\left\{4, \frac{4}{1,2} \mid 6\right\}\right\}$; this is not dualizable, because its facet is not. However, the geometric picture confirms that this apeirotope does actually exist.

Before we pass on to the other vertex-figures, let us eliminate the possibility that we could have $\operatorname{dim} R_{0}=1$ or 3 . These two cases will also be related by $R_{0} \longleftrightarrow R_{0} T$, and so it suffices to consider the latter. Since vert $\left\{4, \frac{4}{1,2} \mid 3\right\} \subset \operatorname{vert}\left\{4, \frac{4}{1,2} \mid 6\right\}$ and, indeed, the symmetry group of the first is a subgroup of that of all the other tori here, it is enough to show that we cannot have $\operatorname{dim} R_{0}=3$ in the case of $\left\{4, \frac{4}{1,2} \mid 3\right\}$. However, in analogy to what we saw in Sect. 6 , this would yield a diagram $\mathcal{A}_{1}(2,3,2, s)$ (in dual form) of an infinite discrete group for some rational $s$, and no such diagram exists.

We now pick up our previous discussion. As we said earlier, we can attempt to adjoin the new generator $R_{0}$ in the other three cases as well. We now have

$$
\left\{\left\{\frac{6}{1,3}, 4: \frac{4}{1,2}\right\},\left\{4, \frac{4}{1,2} \mid 3\right\}\right\} \stackrel{\delta}{\longleftrightarrow}\left\{\left\{4, \frac{4}{1,2} \mid 3\right\},\left\{\frac{4}{1,2}, 6: \frac{4}{1,2}\right\}\right\} .
$$

The first apeirotope is derived in the same way as before from $\mathcal{A}_{2}(3,3)$, and this time (as indicated) there is a dual. We observed in $\left[14\right.$, p. 384] that $\left\{\frac{4}{1,2}, 6: \frac{4}{1,2}\right\}^{\eta}$ degenerates, and so we cannot apply $\eta$ to $\left\{\left\{4, \frac{4}{1,2} \mid 3\right\},\left\{\frac{4}{1,2}, 6: \frac{4}{1,2}\right\}\right\}$. Indeed, if we try, then we obtain facets with triangular faces and planar hexagonal vertex-figures, in other words, planar tilings $\{3,6\}$.

For $\left\{4, \frac{4}{1,2} \mid 6\right\}^{\eta}=\left\{\frac{4}{1,2}, 4: \frac{6}{1,3}\right\}$, applying $\eta$ to $\left\{\left\{\frac{6}{2,3}, 4: \frac{4}{1,2}\right\},\left\{4, \frac{4}{1,2} \mid 6\right\}\right\}$, with the operation $\left(R_{0}, R_{1}, R_{2}, R_{3}\right) \mapsto\left(R_{0}, R_{1} R_{2} R_{1}, R_{3}, R_{2}\right)=:\left(S_{0}, \ldots, S_{3}\right)$, yields

$$
S_{1} S_{0}: z \mapsto(1+\omega y, 1+\omega x),
$$

so that $\left(S_{1} S_{0}\right)^{3}$ is $z \mapsto(y, x)$, which is $R_{2}=S_{3}$. But $\left(S_{1} S_{0}\right)^{3}=S_{3}$ violates the intersection property, and so the corresponding apeirotope does not exist. Actually, 
we know for a different reason that something goes wrong here. Tracing $(1,1)$ under $S_{1} S_{0}$ yields a triangle, and with triangular faces, we would obtain facets of type $\left\{3, \frac{4}{1,2}\right\}$, which is impossible (regular octahedra in $\mathbb{E}^{4}$ can only have planar vertexfigures, see the description in [9] or [20, Sect. 5B] of the realization space of the octahedron). In fact, this triangle is actually a doubly-covered hexagon.

However, $\eta$ does work in the remaining case, and we obtain

$$
\left\{\left\{\frac{6}{1,3}, 4: \frac{4}{1,2}\right\},\left\{4, \frac{4}{1,2} \mid 3\right\}\right\} \stackrel{\eta}{\longrightarrow}\left\{\left\{6, \frac{4}{1,2} \mid \frac{6}{1,2}\right\},\left\{\frac{4}{1,2}, 4: \frac{6}{2,3}\right\}\right\} .
$$

The notation for the facet indicates its type only in the broadest terms (its Petrie polygon is infinite). Because the facet is infinite, the apeirotope can have no (discrete geometric) dual.

We now try to apply the Petrie operation $\pi$ and the central inversion $\kappa$ to these examples. Bearing in mind that $\kappa \eta=\eta$, we see that no more cases will arise to which we can apply $\eta$. Of course, $\pi$ and $\kappa$ commute, and both operations commute with $\tau$ (when it is applicable). As it happens, none of the new examples will be dualizable. Since there is no problem with apeirotopes of the form apeir $Q$, we need only consider those cases with $\operatorname{dim} R_{0}>0$.

Two of the four vertex-figures derived from the tori are self-Petrie, as their notation indicates; thus $\pi$ yields no new apeirotope in these cases. For the other two, we have

$$
\left\{4, \frac{4}{1,2} \mid 6\right\}^{\pi}=\left\{\frac{12}{1,5}, \frac{4}{1,2}: 4,6\right\}, \quad\left\{4, \frac{4}{1,2} \mid 3\right\}^{\pi}=\left\{\frac{6}{1,2}, \frac{4}{1,2}: 4,3\right\} ;
$$

the Petrials are indicated by the notation. We shall say no more about the effects on the corresponding apeirotopes, leaving what details there are to Theorem 4.9, except that their facets must be infinite, since their vertex-figures are four-dimensional.

We now move on to $\kappa$. First, it does not work on $\left\{\left\{6, \frac{4}{1,2} \mid \frac{6}{1,3}\right\},\left\{\frac{4}{1,2}, 4: \frac{6}{2,3}\right\}\right\}$; since $\left\{\frac{4}{1,2}, 4: \frac{6}{2,3}\right\}^{\kappa}=\left\{\frac{4}{1,2}, 4: \frac{6}{1,3}\right\}$, it produces the degenerate example that we have just encountered (this provides another picture of why its face is a doubly-covered triangle). In the remaining cases, we have

$$
\begin{aligned}
& \left\{4, \frac{4}{1,2} \mid 6\right\}^{\kappa}=\left\{\frac{4}{1,2}, \frac{4}{1,2} \mid \frac{6}{1,3}\right\} \cong\{4,4 \mid 6\}, \\
& \left\{4, \frac{4}{1,2} \mid 3\right\}^{\kappa}=\left\{\frac{4}{1,2}, \frac{4}{1,2} \mid \frac{6}{2,3}\right\} \cong\{4,4\}_{6}
\end{aligned}
$$

note that $D_{2}= \pm D_{3}$. By Theorem 4.12, $\left\{\left\{\frac{6}{2,3}, 4: \frac{4}{1,2}\right\},\left\{4, \frac{4}{1,2} \mid 6\right\}\right\}^{\kappa}$ has facets of type $\left\{\frac{6}{0,1}, \frac{4}{1,2}: \frac{4}{0,1}\right\}$, while $\left\{\left\{\frac{6}{1,3}, 4: \frac{4}{1,2}\right\},\left\{4, \frac{4}{1,2} \mid 3\right\}\right\}^{\kappa}$ has facets of type $\left\{\frac{3}{0,1}, \frac{4}{1,2}: \frac{4}{0,1}\right\}$.

Next, referring to $[14$, Sect. 8], we see that $\kappa$ has the same effect as $\pi$ on $\left\{\frac{4}{1,2}, 6: \frac{4}{1,2}\right\}$, which is self-Petrie; thus $\kappa$ preserves $\left\{\left\{4, \frac{4}{1,2} \mid 3\right\},\left\{\frac{4}{1,2}, 6: \frac{4}{1,2}\right\}\right\}$. This may seem to contradict Theorem 4.12; in fact, it merely makes the hole $\{3\}$ of the facet (apparently) doubly-covered.

Finally, under $\pi \kappa=\kappa \pi$, the only cases not yet effectively considered are

$$
\begin{aligned}
& \left\{4, \frac{4}{1,2} \mid 6\right\}^{\pi \kappa}=\left\{\frac{12}{1,5}, \frac{4}{1,2}: \frac{4}{1,2}, \frac{6}{1,3}\right\}, \\
& \left\{4, \frac{4}{1,2} \mid 3\right\}^{\pi \kappa}=\left\{\frac{6}{2,3}, \frac{4}{1,2}: \frac{4}{1,2}, \frac{6}{2,3}\right\} .
\end{aligned}
$$


We leave the determination of the faces of the resulting apeirotopes (using Theorem 4.12 on top of Theorem 4.9) to the interested reader.

Under $\varsigma$, we have

$$
\begin{aligned}
& \left\{4, \frac{4}{1,2} \mid 3\right\} \mapsto\left\{\frac{6}{1,2}, 3: \frac{6}{1,2}\right\}, \\
& \left\{4, \frac{4}{1,2} \mid 4\right\} \mapsto\left\{\frac{8}{1,3}, 4: \frac{8}{1,3}\right\}, \\
& \left\{4, \frac{4}{1,2} \mid 6\right\} \mapsto\left\{\frac{12}{1,5}, 6: \frac{12}{1,5}\right\} ;
\end{aligned}
$$

as pointed out in [14], 5 degenerates on tori of type $\left\{\frac{4}{1,2}, 4\right\}$. We have included the additional case here, because they all exhibit the same behaviour. Observe that $\left\{\frac{6}{1,2}, 3: \frac{6}{1,2}\right\} \cong\{6,3\}_{(1,1)}$ is also a torus. Following through $\sigma$ (rather than $\varsigma$ ) on the original generators, we can take the new generators to be

$$
\begin{aligned}
& R_{1}: z \mapsto(x, y), \\
& R_{2}: z \mapsto(\bar{x}, \varphi \bar{y}), \\
& R_{3}: z \mapsto(\bar{x}, \bar{y}),
\end{aligned}
$$

where $\varphi=\omega, i$, or $-\bar{\omega}=-\omega^{2}$ according as $r=3,4$, or 6 , indicating the holes of the original tori or the vertex-figures of the new polyhedra. Each polyhedron has vertexset $\mathcal{U}_{r}:=\left\{\left(\varphi^{n}, 0\right),\left(0, \varphi^{n}\right) \mid 0 \leqslant n<r\right\}$; the initial vertex is $(1,0)$, which is joined to $(0,1)$ by the initial edge. Further, define the reflexion $T$ by

$$
T: z \mapsto(x,-y)
$$

For $r=4$ or 6 , we see that $T=\left(R_{2} R_{3}\right)^{r / 2}$ is as we have generally defined it; it is convenient to extend the notation to the case $r=3$ as well.

For each of these $r$, let $s$ be given by

$$
\frac{1}{s}+\frac{1}{r}=\frac{1}{2}
$$

and let $M_{r}:=\{s, r\}$, the tiling of the plane $\mathbb{E}^{2}$ by regular $s$-gons. It turns out that the vertex- and edge-set of each apeirotope for $r$ must be those of the product $M_{r} \times M_{r}$. Indeed, for each $r$, we have

$$
\begin{aligned}
z & \mapsto(1-x,-y), \\
z & \mapsto(1-\bar{x},-y), \\
z & \mapsto(1-x, y), \\
z & \mapsto(1-\bar{x}, y),
\end{aligned}
$$

according as $\operatorname{dim} R_{0}=0,1,2$, or 3 . Clearly, the first and third, and second and fourth, are related by $\tau: R_{0} \mapsto R_{0} T$. Now it is obvious that each of these reflexions is compatible with the decomposition $\mathbb{E}^{4}=\mathbb{E}^{2} \oplus \mathbb{E}^{2}$, and only a little thought is 
needed to show that the resulting vertex- and edge-sets are as claimed. For the first and third, the face is of type $\left\{\frac{2}{0,1}\right\}$, while for the second and fourth, it is $\{4\}$ (such a square is the product of an edge of each of the components $M_{r}$, and all such products are faces). The vertex-figures are self-Petrie; the Petrie operation $\pi$ in any case does not change the type of the face. Finally, $\kappa$ preserves $\left\{\frac{12}{1,5}, 6: \frac{12}{1,5}\right\}$ and $\left\{\frac{6}{1,2}, 3: \frac{6}{1,2}\right\}$ (actually, it sends the latter into a congruent copy).

However, two of the resulting apeirotopes degenerate, namely, those with $r=6$ and $\operatorname{dim} R_{0}$ odd. To see this, note that $R_{0}$ will take the initial vertex-figure into its enantiomorph, reversing the twist of its helical faces. Since there are odd edge-circuits \{3\} (the faces of $M_{6}$ ), two enantiomorphic vertex-figures will try to occur at the same vertex, which is impossible.

\section{The Case $\mathcal{P}_{5} \rtimes \mathcal{D}_{5}$}

We now look at apeirotopes whose groups are subgroups of $\mathcal{P}_{5} \rtimes \mathcal{D}_{5}$. Here, we find it convenient to work in the hyperplane

$$
E:=\left\{x=\left(\xi_{0}, \ldots, \xi_{4}\right) \in \mathbb{E}^{5} \mid \xi_{0}+\cdots+\xi_{4}=0\right\} .
$$

In particular, all our polytopes will have vertices in the lattice $\Lambda:=E \cap \mathbb{Z}^{5}$, whose generators are the $e_{0}-e_{j}$ for $j=1, \ldots, 4$, where $\left\{e_{0}, \ldots, e_{4}\right\}$ is the standard basis of $\mathbb{E}^{5}$. We can identify the symmetry group $[3,3,3]$ of the 4 -simplex $\{3,3,3\}$ with the permutation group $\mathcal{S}_{5}$ acting on the standard basis; it is useful to note that the (linear) reflexions in $\mathcal{S}_{5} \rtimes \mathcal{C}_{2}=\mathcal{S}_{5} \times \mathcal{C}_{2}$ are composed of one or two (disjoint) transpositions, together with $\pm I$.

We begin by investigating the apeirotopes with blended vertex-figures. Since we do not have ordinary 4-fold rotations in the group, the only possibilities are $\{3,3\} \#\{2\}$ and $\{4,3\} \#\{2\}$. Moreover, the (false) cluster polytope must be $\{3,3,3\}$ or $\{3,3,3\}\}^{\zeta}$.

The results of Sect. 6 tell us how to calculate the related apeirotopes. We have



The facets of the apeirotopes in the top row are the universal $\{6,6 \mid 3\}$ and $\{6,4 \mid 3\}$. The facet of the bottom middle apeirotope is $\{\infty, 6\}_{4,4} \#\{\infty\}$, using the notation of [19] (see also Remark 4.11); all its holes and zigzags are infinite, which accounts for the truncated notation. Finally, the facet at bottom right is $\left\{\frac{5}{1,2}, \frac{4}{1,2} \mid \frac{6}{1,3}\right\}^{\pi}$, where $\left\{\frac{5}{1,2}, \frac{4}{1,2} \mid \frac{6}{1,3}\right\} \cong\{5,4 \mid 6\}$ is universal (bear in mind Remark 4.2 about holes and 2-zigzags in this case).

Apart from the blended one, there are three basic vertex-figures in this family of apeirotopes; all others will have the same or (in the last case) closely related vertexsets. We shall take for granted that we can form the free abelian apeirotope apeir $Q$ on each vertex-figure $Q$, and so these will receive little mention. 
Comparing [14, p. 368], we see that we can take the group $\mathcal{G}_{0}=\mathcal{S}_{5} \rtimes \mathcal{C}_{2}=\mathcal{S}_{5} \times \mathcal{C}_{2}$ of $Q_{1}:=\left\{4, \frac{6}{1,3} \mid 3\right\} \cong\{4,6 \mid 3\}$ (found by Coxeter [3]) to have generators

$$
\begin{aligned}
& R_{1}: x \mapsto\left(\xi_{1}, \xi_{0}, \xi_{2}, \xi_{3}, \xi_{4}\right), \\
& R_{2}: x \mapsto-\left(\xi_{4}, \ldots, \xi_{0}\right), \\
& R_{3}: x \mapsto\left(\xi_{0}, \xi_{1}, \xi_{3}, \xi_{2}, \xi_{4}\right)
\end{aligned}
$$

The initial vertex is $v_{1}=(1,0,0,0,-1)$, the initial edge joins $v_{1}$ to $(0,1,0,0,-1)$, and the whole vertex-set is $V_{1}:=v_{1} \mathcal{S}_{5}$, giving 20 vertices in all. Thus the ratio of edge-length to circumradius is 1 , indicating that $\left\{4, \frac{6}{1,3} \mid 3\right\}$ is a suitable vertex-figure for an apeirotope with triangular faces. Note further that its Petrie polygon is of type $\left\{\frac{10}{1,3}\right\}$; indeed, $\left(R_{1} R_{2} R_{3}\right)^{5}=-I$.

In fact, we have here

$$
T=\left(R_{2} R_{3}\right)^{3}: x \mapsto-\left(\xi_{4}, \xi_{1}, \xi_{2}, \xi_{3}, \xi_{0}\right)
$$

we readily see that

$$
R_{0}: x \mapsto v_{1}+\left(\xi_{4}, \xi_{1}, \xi_{2}, \xi_{3}, \xi_{0}\right)=\left(1+\xi_{4}, \xi_{1}, \xi_{2}, \xi_{3},-1+\xi_{0}\right)
$$

is a hyperplane reflexion such that $R_{0} T$ is the point-reflexion for apeir $Q_{1}$. We thus obtain the apeirotope $P_{1}:=\left\{\{3,4\},\left\{4, \frac{6}{1,3} \mid 3\right\}\right\}$. It is straightforward to see that the common vertex-set of these apeirotopes is exactly $\Lambda$ and that their edges join all pairs of nearest points of $\Lambda$ (at distance $\sqrt{2}$ ). Moreover, it should be clear that $P_{1}$ is dualizable; its dual $\left\{\left\{6, \frac{4}{1,2} \mid 3\right\},\left\{\frac{4}{1,2}, 3\right\}\right\}$ is that with cluster polytope $\{3,3,3\}$ that we have just described. As we pointed out in Sect. 6, this latter apeirotope is derivable from a diagram $\mathcal{A}_{1}$ as in Fig. 1 -in this case, $\mathcal{A}_{1}(3,3,2,3\}$. In a sense (as we shall see shortly), Theorem 6.5 actually tells us more than we need to know about the facet $\left\{6, \frac{4}{1,2} \mid 3\right\}$.

We next consider the effects of $\kappa, \pi$ and $\eta$. We shall deal with $\varsigma$ separately at the end, since it does not fit in with the pattern of the rest. Under $\kappa:\left(R_{1}, R_{2}, R_{3}\right) \mapsto$ $\left(-R_{1}, R_{2}, R_{3}\right)=:\left(S_{1}, S_{2}, S_{3}\right)$, we have

$$
\left\{4, \frac{6}{1,3} \mid 3\right\}^{\kappa}=\left\{\frac{4}{1,2}, \frac{6}{1,3}: \frac{5}{1,2}\right\} \cong\{4,6\}_{5} .
$$

This isomorphism may initially seem somewhat surprising. However, $Q_{1}^{\kappa}$ and $Q_{1}$ have the same vertex-set $V_{1}$ and thus the same group $\mathcal{S}_{5} \times \mathcal{C}_{2}$; since the period of $S_{1} S_{2} S_{3}$ is now 5, the new polyhedron is a quotient of the universal $\{4,6\}_{5}$ with the same group (see [4, Table 8]), and so is isomorphic to it. (Note, by contrast, that the new hole is $\left\{\frac{6}{1,3}\right\}$.)

We then have

$$
\left\{\{3,4\},\left\{4, \frac{6}{1,3} \mid 3\right\}\right\}^{\kappa}=\left\{\left\{6, \frac{4}{1,2} \mid 4\right\},\left\{\frac{4}{1,2}, \frac{6}{1,3}: \frac{5}{1,2}\right\}\right\},
$$

where straightforward calculations (compare Sect. 6) show that the facet is the universal Petrie-Coxeter apeirohedron $\{6,4 \mid 4\}$. 
Remark 8.1 The interchange

$$
\{4,6 \mid 3\} \stackrel{\kappa}{\longleftrightarrow}\{4,6\}_{5}
$$

as abstract regular polyhedra is of interest in its own right. With their groups $\left\langle R_{1}, R_{2}, R_{3}\right\rangle$ and $\left\langle S_{1}, S_{2}, S_{3}\right\rangle$ as above, we see that $\left(S_{1} S_{2} S_{3} S_{2}\right)^{3}=-I$ is also the central involution, and the abstract change of generators is effected by

$$
S_{1}=\left(R_{1} R_{2} R_{3}\right)^{5} R_{1}, \quad R_{1}=\left(S_{1} S_{2} S_{3} S_{2}\right)^{3} S_{1} .
$$

This is easily checked. Since $R_{j}=S_{j}$ for $j=2,3$, we have

$$
\begin{aligned}
& S_{1} S_{2} S_{3}=\left(R_{1} R_{2} R_{3}\right)^{6} \quad \Longrightarrow \quad\left(S_{1} S_{2} S_{3}\right)^{5}=\left(R_{1} R_{2} R_{3}\right)^{30}=I, \\
& R_{1} R_{2} R_{3} R_{2}=\left(S_{1} S_{2} S_{3} S_{2}\right)^{4} \Longrightarrow\left(R_{1} R_{2} R_{3} R_{2}\right)^{3}=\left(S_{1} S_{2} S_{3} S_{2}\right)^{12}=I \text {, }
\end{aligned}
$$

so that the defining relations for the group of one imply those of the other.

Since the facets and vertex-figures of these two apeirotopes are universal, it is natural to ask about the apeirotopes themselves.

Theorem 8.2 The following apeirotopes are universal:

$$
\begin{aligned}
&\left\{\{3,4\},\left\{4, \frac{6}{1,3} \mid 3\right\}\right\} \cong\{\{3,4\},\{4,6 \mid 3\}\}, \\
&\left\{\left\{6, \frac{4}{1,2} \mid 4\right\},\left\{\frac{4}{1,2}, \frac{6}{1,3}: \frac{5}{1,2}\right\}\right\} \cong\left\{\{6,4 \mid 4\},\{4,6\}_{5}\right\} .
\end{aligned}
$$

Proof In each case, we appeal to the circuit criterion of [19, Theorem 2.5] (see also [20, Theorem 2F4]), which states that the group of a regular polytope is determined by that of its vertex-figure and the relations induced by its edge-circuits. Here, we can also take the group of the facet for granted. In the present case, the edges join adjacent vertices of the lattice $\Lambda$, and edge-circuits can clearly be contracted over the triangles formed by these edges. The first case is then obvious: each triangle is equivalent to the basic face. The other is nearly as obvious. This time, we regard a triangle as a hole in the original vertex-figure $\left\{4, \frac{6}{1,3} \mid 3\right\}$. However, this vertex-figure and $\left\{\frac{4}{1,2}, \frac{6}{1,3}: \frac{5}{1,2}\right\}$ have the same group, and so the relation determining the hole is implicit in that group. Thus, in each case, no relations are needed to specify the group other than those arising from the facet and vertex-figure, and this completes the proof.

Remark 8.3 The first case (and that of the next Theorem 8.4) is treated in [18, Sect. 7], where the universality is a specific consequence of the construction from a diagram $\mathcal{A}_{1}$ with integer marks on its branches.

Because of its triangular faces, we cannot apply $\pi$ to $\left\{\{3,4\},\left\{4, \frac{6}{1,3} \mid 3\right\}\right\}$. However, we can to the other, and we obtain $\left\{\left\{6, \frac{5}{1,2} \mid 4\right\},\left\{\frac{5}{1,2}, \frac{6}{1,3}: \frac{4}{1,2}\right\}\right\}$. Calculations show that the facet is $\left\{6, \frac{5}{1,2} \mid 4\right\} \cong\{6,5 \mid 4\}$ and that the vertex-figure is $\left\{\frac{5}{1,2}, \frac{6}{1,3}: \frac{4}{1,2}\right\} \cong$ $\{5,6\}_{4}$. It is possible that this apeirotope is the universal $\{\{6,5 \mid 4\},\{5,6\} 4\}$, but if so, 
it is far from obvious. If its group is $\left\langle T_{0}, \ldots, T_{3}\right\rangle$, then geometrically we obtain the standard generators of $\mathcal{P}_{5}$ as $\left(T_{0}, T_{1} T_{0} T_{1}, T_{2} T_{3} T_{2}, T_{3}, T_{2} T_{1} T_{0} T_{1} T_{2}\right)=\left(R_{0}, \ldots, R_{4}\right)$, but the abstract isomorphism depends on the deep hole of the universal apeirotope being $\{3\}$; in turn, we can describe the geometric apeirotope abstractly as

$$
\left\{\left\{6, \frac{5}{1,2} \mid 4\right\},\left\{\frac{5}{1,2}, \frac{6}{1,3}: \frac{4}{1,2}\right\}\right\} \cong\left\{\{6,5 \mid 4\},\{5,6\}_{4} \mid 3\right\}
$$

in the notation of Sect. 4 (it is thus open to question whether the final entry, denoting the deep hole, is needed). We do know that we cannot apply $\kappa$ to it, because we cannot apply $\pi$ to $\left\{\{3,4\},\left\{4, \frac{6}{1,3} \mid 3\right\}\right\}$.

There remains the application of $\eta$. First, $Q_{1}^{\eta}=\left\{\frac{6}{1,3}, 6: \frac{6}{2,3}\right\}$ (see [14, p. 384]) with the same vertex-set $V_{1}$ (because its holes $\{3\}$ give $Q_{1}$ odd edge-circuits) and hence the same group $\mathcal{S}_{5} \times \mathcal{C}_{2}$. Theorem 4.15 tells us that (assuming its existence) $\left\{\{3,4\},\left\{4, \frac{6}{1,3} \mid 3\right\}\right\}^{\eta}$ has facets the vertex-figures of $P$, namely, $Q_{1}=\left\{4, \frac{6}{1,3} \mid 3\right\}$, and thus $P_{1}^{\eta}$ is of the form $\left\{\left\{4, \frac{6}{1,3} \mid 3\right\},\left\{\frac{6}{1,3}, 6: \frac{6}{2,3}\right\}\right\}$. Since $Q_{1}^{\eta}$ is certainly not the universal $\{6,6\}_{6}$ (a presentation of its group will necessarily be rather complicated), the universality of $P_{1}^{\eta}$ is also questionable.

Of course, we are now in a position to apply $\kappa$ and $\pi$ again. (Since $\kappa \eta=\eta$, there are no more examples yet to which $\eta$ can be applied.) In fact, both $\kappa$ and $\pi$ have the same effect and result in

$$
\left\{\left\{4, \frac{6}{1,3} \mid 3\right\},\left\{\frac{6}{1,3}, 6: \frac{6}{2,3}\right\}\right\}^{\pi}=\left\{\left\{4, \frac{6}{2,3}: \frac{5}{1,2} \mid 3\right\},\left\{\frac{6}{2,3}, 6: \frac{6}{1,3}\right\}\right\} .
$$

We shall meet this new facet again later, where we will justify the notation for it.

Summarizing what we have so far, we have the following picture of further connexions among our apeirotopes (we repeat that we also have apeir $Q$ for each vertexfigure $Q$ in the diagram). These give all vertex-figures with vertex-set $V_{1}$, except for two derived indirectly by $\varsigma$ that we deal with at the end of the section.

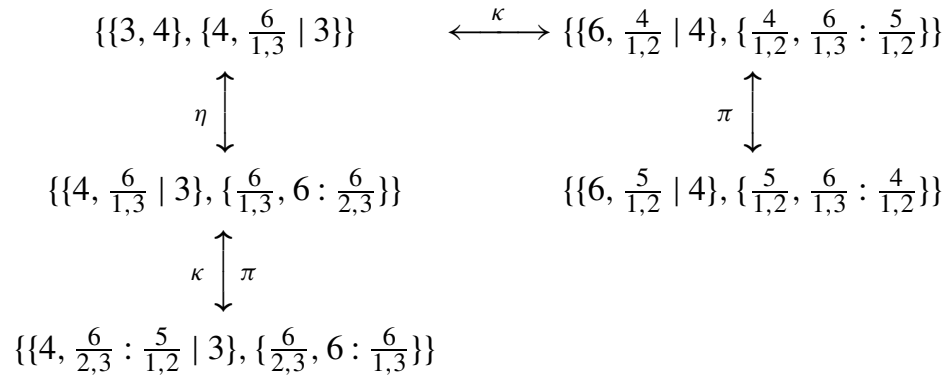

Among these apeirotopes, only $\left\{\{3,4\},\left\{4, \frac{6}{1,3} \mid 3\right\}\right\}$ and $\left\{\left\{4, \frac{6}{1,3} \mid 3\right\},\left\{\frac{6}{1,3}, 6: \frac{6}{2,3}\right\}\right\}$ have duals; $\left\{\left\{4, \frac{6}{2,3}: \frac{5}{1,2} \mid 3\right\},\left\{\frac{6}{2,3}, 6: \frac{6}{1,3}\right\}\right\}$ has finite facets and vertex-figures, but neither is dualizable. The facet of the first dual is the dual $\left\{6, \frac{4}{1,2} \mid 3\right\}$ of $\left\{4, \frac{6}{1,3} \mid 3\right\}$, which is also the vertex-figure of the second. Centred at $o$, it has initial vertex $v_{2}=$ $(1,1,0,-1,-1)$ with the initial edge joining $v_{2}$ to $(1,1,-1,0,-1)$, and vertex-set $V_{2}:=v_{2} \mathcal{S}_{5}$, so that there are 30 vertices in all.

We can now take the facets of the apeirotope $\left\{\left\{6, \frac{4}{1,2} \mid 3\right\},\left\{\frac{4}{1,2}, 3\right\}\right\}$ to be the translates of this initial one with vertex-set $V_{2}$ by $2 \Lambda$. (This is the first case with blended 
vertex-figure; the cluster polytope is a simplex with edge-length $\sqrt{2}$.) We have already seen the effect of $\pi$ on this. As we might suspect, we have

Theorem 8.4 There are two universal regular 4-apeirotopes

$$
\begin{aligned}
&\left\{\left\{6, \frac{4}{1,2} \mid 3\right\},\left\{\frac{4}{1,2}, 3\right\}\right\} \cong\{\{6,4 \mid 3\},\{4,3\}\}, \\
&\left\{\left\{6, \frac{6}{1,3} \mid 3\right\},\left\{\frac{6}{1,3}, 3: \frac{4}{1,2}\right\}\right\} \cong\left\{\{6,6 \mid 3\},\{6,3\}_{4}\right\} .
\end{aligned}
$$

Proof The universality of the first is guaranteed by the fact that it is the dual of the universal apeirotope $\left\{\{3,4\},\left\{4, \frac{6}{1,3} \mid 3\right\}\right\} \cong\{\{3,4\},\{4,6 \mid 3\}\}$. For the second, we merely observe that its faces and holes of facets are the same as those of the first (these hexagons and triangles form quasi-regular planar tessellations). Any contraction of an edge-circuit in the first therefore gives a corresponding contraction in the second, which is all that we need.

Now let us take $\left\{6, \frac{4}{1,2} \mid 3\right\}$ as a vertex-figure, naturally centred at $o$. In this case (with the previous generators in reverse order), $T=\left(R_{2} R_{3}\right)^{2}$ is

$$
T: x \mapsto\left(\xi_{1}, \xi_{0}, \xi_{2}, \xi_{4}, \xi_{3}\right)
$$

Applying the corresponding operation $\tau$ to the point-reflexion for apeir $\left\{6, \frac{4}{1,2} \mid 3\right\}$, we arrive at the generators

$$
\begin{aligned}
& R_{0}: x \mapsto\left(1-\xi_{1}, 1-\xi_{0},-\xi_{2},-1-\xi_{4},-1-\xi_{3}\right), \\
& R_{1}: x \mapsto\left(\xi_{0}, \xi_{1}, \xi_{3}, \xi_{2}, \xi_{4}\right), \\
& R_{2}: x \mapsto-\left(\xi_{4}, \ldots, \xi_{0}\right), \\
& R_{3}: x \mapsto\left(\xi_{1}, \xi_{0}, \xi_{2}, \xi_{3}, \xi_{4}\right) .
\end{aligned}
$$

Since $\operatorname{dim} T=2$, we see that $\operatorname{dim} R_{0}=2$ also. In view of this, we should not be at all surprised to discover that the resulting apeirotope is

$$
\left\{\left\{\frac{6}{1,3}, 6: \frac{6}{2,3}\right\},\left\{6, \frac{4}{1,2} \mid 3\right\}\right\}=\left\{\left\{4, \frac{6}{1,3} \mid 3\right\},\left\{\frac{6}{1,3}, 6: \frac{6}{2,3}\right\}\right\}^{\delta} .
$$

Bear in mind (see [14, p. 272]) that its facet $\left\{\frac{6}{1,3}, 6: \frac{6}{2,3}\right\}$ is self-dual.

This is now the jumping-off point for applying $\kappa$ and $\pi$. Without going into detail, there results

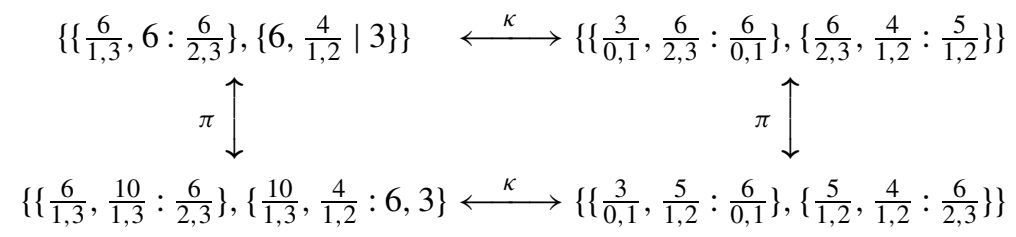

Apart from the apeirotopes apeir $Q$, these are all those whose vertex-figures have vertex-set $V_{2}$. 
The vertex-figures of the two apeirotopes on the right are of interest; they are (abstractly) the Petrie pair $\{6,4\}_{5}$ and $\{5,4\}_{6}$. As with the previous isomorphisms $\left\{\frac{4}{1,2}, \frac{6}{1,3}: \frac{5}{1,2}\right\} \cong\{4,6\}_{5}$ and $\left\{\frac{5}{1,2}, \frac{6}{1,3}: \frac{4}{1,2}\right\} \cong\{5,6\}_{4}$, these isomorphisms follow from the fact that the geometric polyhedra have the same group $\mathcal{S}_{5} \times \mathcal{C}_{2}$ satisfying the same relations as the abstract ones.

Remark 8.5 Observe that, even though $\left\{\frac{4}{1,2}, \frac{6}{1,3}: \frac{5}{1,2}\right\}$ and $\left\{\frac{6}{2,3}, \frac{4}{1,2}: \frac{5}{1,2}\right\}$ are dual polyhedra, their groups are not obtained by the usual reversal $\left(R_{1}, R_{2}, R_{3}\right) \leftrightarrow$ ( $R_{3}, R_{2}, R_{1}$ ) of the generators of their groups. In fact, what we have done shows that the duality is actually induced by $\left(R_{1}, R_{2}, R_{3}\right) \leftrightarrow\left(-R_{3}, R_{2},-R_{1}\right)$ (which is not even conjugate to the reversal of the order of the generators).

The final basic vertex-figure in this family is $\left\{4, \frac{6}{2,3}: \frac{5}{1,2} \mid 3\right\}=\left\{4, \frac{6}{1,3} \mid 6\right\}^{\kappa_{2}}$, whose group is just $\mathcal{S}_{5}$. We therefore replace the generator $R_{2}$ in the group of $\left\{4, \frac{6}{1,3} \mid 3\right\}$ by $-R_{2}$, so that, after relabelling, the new generator is

$$
R_{2}: x \mapsto\left(\xi_{4}, \ldots, \xi_{0}\right)
$$

The initial vertex is $v_{3}=(3,-2,-2,-2,3)$, the initial edge joins $v_{3}$ to $(-2,3,-2$, $-2,3)$, and the set $V_{3}:=v_{3} \mathcal{S}_{5}$ of its 10 vertices consists of the mid-points of the edges of a regular 4-simplex. To justify the notation for this polyhedron, observe that it is obtained by identifying pairs of opposite vertices of $\left\{4, \frac{6}{1,3} \mid 3\right\}$ or $\left\{\frac{4}{1,2}, \frac{6}{1,3}: \frac{5}{1,2}\right\}$; indeed, we can map $\pm v_{1}$ to $v_{3}$ and complete the identification by applying $\mathcal{S}_{5}$. This identification is by $-I$, which halves the lengths of the Petrie polygons of the first and the holes of the second.

In this case,

$$
T=\left(R_{2} R_{3}\right)^{3}: x \mapsto\left(\xi_{4}, \xi_{1}, \xi_{2}, \xi_{3}, \xi_{0}\right),
$$

so that, applying the operation $\tau$ to the point-reflexion for apeir $\left\{4, \frac{6}{2,3}: \frac{5}{1,2} \mid 3\right\}$, we obtain

$$
R_{0}: x \mapsto\left(3-\xi_{4},-2-\xi_{1},-2-\xi_{2},-2-\xi_{3}, 3-\xi_{0}\right),
$$

a reflexion of dimension 1 . The corresponding apeirotope is $\left\{\left\{\frac{6}{1,3}, 4: \frac{6}{1,3}\right\},\left\{4, \frac{6}{2,3}\right.\right.$ : $\left.\left.\frac{5}{1,2} \mid 3\right\}\right\}$. Its facet is isomorphic to the universal $\{6,4\}_{6}$; it is, in fact, $\{6,4\}_{6} \#\{2\}$ (note that the hyperplane $\xi_{2}=-1$ is fixed by each of $R_{0}, R_{1}$, and $R_{2}$ and that the vertices of the facet lie in $\xi_{2}=0$ or -2 ). Of course, this apeirotope is not dualizable, since its facet is infinite and its vertex-figure has no dual.

We cannot apply $\eta$ to $\left\{4, \frac{6}{2,3}: \frac{5}{1,2} \mid 3\right\}$; the result degenerates (see [14]) because its hexagonal vertex-figure would collapse to a doubly-covered triangle.

Remark 8.6 In fact, $\left\{4, \frac{6}{2,3}: \frac{5}{1,2} \mid 3\right\}^{\eta}$ does not even cover a regular polyhedron under this collapse. This polyhedron would be a torus of type $\{6,3\}$; such tori have $2 s^{2}$ or $6 s^{2}$ vertices for some $s$, and 10 is not of this form.

However, we can apply both $\pi$ and $\kappa$; note that $\kappa$ doubles the number of vertices of the vertex-figures, because $\left\{4, \frac{6}{2,3}: \frac{5}{1,2} \mid 3\right\}$ is not centrally symmetric. What we 
obtain is then depicted in the following diagram; we have not given details of the facets of the last two, since we know little about them other than that they are infinite.

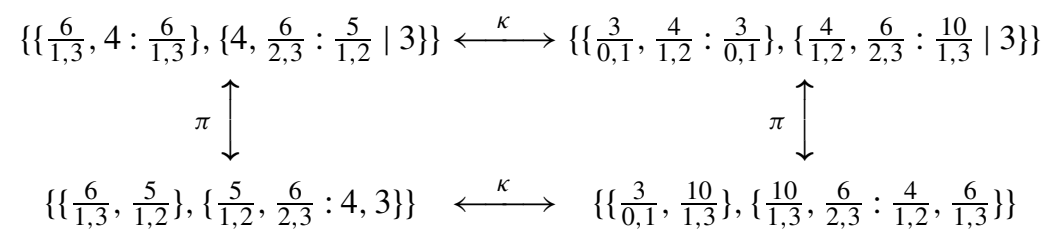

We finally come to the applications of $\varsigma$; since they yield the dodecahedron and some relatives, these are potentially interesting. The pattern of relationships is pictured in the diagram:

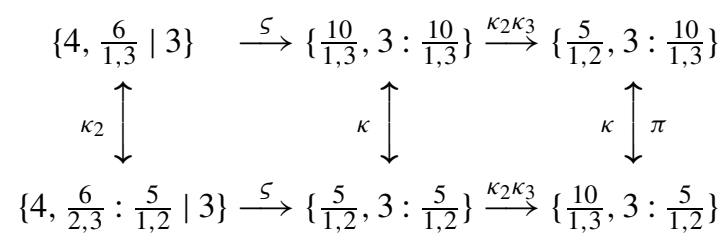

We shall not go into too many details for reasons that will emerge. The polyhedron $\left\{\frac{5}{1,2}, 3: \frac{5}{1,2}\right\}$ is the hemi-dodecahedron $\{5,3\}_{5}$, whose edge-graph is the famous Petersen graph $G(5,2)$; we can take its vertex-set to be $V_{3}$, with initial vertex (if we follow the effect of $\varsigma$ on the original generators) $(3,3,-2,-2,-2)$. (See [7] for the complete classification of the cubic Petersen graphs $G(p, k)$ with $2 p$ vertices and [10] for the fact that each is the edge-graph of a regular polyhedron of type $\{p, 3\}$.) Next, $\left\{\frac{10}{1,3}, 3: \frac{10}{1,3}\right\}$ is a double cover of it (different from the dodecahedron), whose edge-graph is the Petersen-type graph $G(10,3)$ with the same initial vertex $(3,3,-2,-2,-2)$ and whole vertex-set $\pm V_{3}$. Both these polyhedra are self-Petrie or, rather, the Petrial pairs are enantiomorphs with helical faces of opposite twists.

Since the vertex-figure of these two polyhedra is $\{3\}$, there is no corresponding reflexion $T$. Nevertheless, we can try to replace the point-reflexion of the free abelian apeirotope by

$$
R_{0}: x \mapsto\left(3-\xi_{1}, 3-\xi_{0},-2-\xi_{2},-2-\xi_{3},-2-\xi_{4}\right),
$$

which does commute with $R_{2}$ and $R_{3}$, just as if $T$ did exist. However, there are odd edge-circuits: go successively in cyclic permutations of the direction $(3,3,-2,-2,-2)$. Because $\operatorname{dim} R_{0}=1$ is odd, such a circuit changes the initial vertex-figure into its enantiomorph; we thus have a contradiction, and the resulting apeirotope degenerates.

If we now apply $\kappa_{2} \kappa_{3}$ (that is, change the signs of both $R_{2}$ and $R_{3}$ ), then we obtain the dodecahedron $\left\{\frac{5}{1,2}, 3: \frac{10}{1,3}\right\} \cong\{5,3\}$ and its Petrial $\left\{\frac{10}{1,3}, 3: \frac{5}{1,2}\right\}$. Each has initial vertex $(1,-1,0,0,0)$ and vertex-set $V_{1}$; of course, they have the same edges as well. Once again, we do not have a reflexion $T$, but we can act as if we did, and introduce the hyperplane reflexion

$$
R_{0}: x \mapsto\left(1+\xi_{1},-1+\xi_{0}, \xi_{2}, \xi_{3}, \xi_{4}\right)
$$


As before, though, there are odd edge-circuits (triangles here), and since $\operatorname{dim} R_{0}=$ 3 is odd, we have a clash of enantiomorphic vertex-figures, and so the apeirotope degenerates.

In a way, this is unfortunate, since the facet of the first of these latter two would be $\left\{4, \frac{5}{1,2} \mid 6\right\} \cong\{4,5 \mid 6\}$, the universal apeirotope with group $\mathcal{P}_{5} \rtimes \mathcal{D}_{5}$. The operation $\varphi_{2}$ of [19] (see also [20, p. 194]) interchanges face and hole here, and results in $\left\{6, \frac{5}{1,2} \mid\right.$ $4\} \cong\{6,5 \mid 4\}$ (which we have already encountered as the facet of the apeirotope $\left.\left\{\left\{6, \frac{5}{1,2} \mid 4\right\},\left\{\frac{5}{1,2}, \frac{6}{1,3}: \frac{4}{1,2}\right\}\right\}\right)$.

Remark 8.7 It is of interest to note that, if we blend these degenerate examples (and the two similar cases at the end of Sect. 7) with a segment or an apeirogon, then they become polytopal, that is, non-degenerate. Compare here Remark 5.1.

In conclusion, then, in $\mathbb{E}^{4}$ we only have the free abelian apeirotopes apeir $Q$ for these pentagonal vertex-figures $Q$.

Remark 8.8 We cannot change the sign of $R_{2}$ (or $R_{3}$ ) alone; the group $\left\langle-R_{2}, R_{3}\right\rangle$ has $o$ as its only fixed point. In [14] we analysed these polyhedra using quaternions, and the reason for the exclusion of this change of sign emerged from the general theory.

Returning to the vertex-figures, since $\left\{4, \frac{6}{2,3}: \frac{5}{1,2} \mid 3\right\}$ and $\left\{\frac{5}{1,2}, 3: \frac{5}{1,2}\right\}$ have the same vertex-set $V_{3}$, it follows that we can regard the latter as inscribed in the former. In fact, it is so inscribed in two ways, which are the Petrials of each other. Similarly, $\left\{4, \frac{6}{1,3} \mid 3\right\}$ and $\left\{\frac{5}{1,2}, 3: \frac{10}{1,3}\right\}$ have the same vertex-set $V_{1}$, and so the latter is inscribed in the former. Again, this is in two ways; the other can be thought of as $\left\{\frac{5}{1,2}, 3\right.$ : $\left.\frac{10}{1,3}\right\}^{\pi \kappa}$, since the double operation $\pi \kappa=\kappa \pi$ produces an enantiomorphic copy of the original with the same vertices. Naturally, we then have corresponding apeirotopes inscribed in one another, but only those resulting from the "apeir" construction.

\section{The Case $\mathcal{U}_{5}$}

Before we begin the actual classification in the last case, we recall two approaches and introduce yet a third. If the vertex-figure is blended, then (up to Petriality) we may take it to be of the form $Q \#\{2\}$, where $Q$ is one of the crystallographic polyhedra $\{3,3\},\{3,4\},\{4,3\}$. Referring to Sect. 6 , we saw that the cases where $\operatorname{dim} R_{0}=0$ or 2 lead to blends. Otherwise, by applying the operation $\zeta$ to the cluster polytope $C$ if necessary, we can assume that $\operatorname{dim} R_{0}=3$; we have seen that this leads to some diagram $\mathcal{A}_{1}$ of a group generated by hyperplane reflexions twisted by an automorphism.

For the third approach, comparing [14, 17], we see that, if $\left\langle S_{0}, \ldots, S_{4}\right\rangle$ is the group of an ordinary (classical) regular 5-apeirotope in $\mathbb{E}^{4}$, then the mixing operation

$$
\left(S_{0}, \ldots, S_{4}\right) \mapsto\left(S_{1}, S_{0} S_{2}, S_{3}, S_{4}\right)=:\left(R_{0}, \ldots, R_{3}\right)
$$


yields the group $\mathcal{G}(P)=\left\langle R_{0}, \ldots, R_{3}\right\rangle$ of a four-dimensional regular apeirotope $P$ of rank 4. Recalling the definition of the diagrams $\mathcal{A}_{1}$ in Sect. 6, the analysis of [14] shows that

$$
\begin{aligned}
&\{4,3,3,4\} \mapsto \mathcal{A}_{1}\left(3,3, \frac{3}{2}, 4\right), \\
&\{3,4,3,3\} \mapsto \mathcal{A}_{1}\left(3,4, \frac{3}{2}, 3\right), \\
&\{3,3,4,3\} \mapsto \mathcal{A}_{1}(2,4,2,3) .
\end{aligned}
$$

In the last case, the mixing leads to a proper subgroup $[4,3,3,4] \rtimes \mathcal{C}_{2}$. However, in the other two cases, when $S_{2} S_{3}$ is of period 3, we see that

$$
\left(R_{1} R_{2}\right)^{3}=\left(S_{0} S_{2} S_{3}\right)^{3}=S_{0}, \quad S_{0} R_{1}=S_{2},
$$

and so we can recover the whole group. Notice that, as we observed in Remark 6.8, because the vertex-figure is blended in each case, we cannot apply the operators $\kappa$ or (in the first case) $\eta$.

Remark 9.1 The apeirotope dealt with in Sect. 8, which we alternatively derived from the diagram $\mathcal{A}_{1}(3,3,2,3)$, is not obtainable by such a mixing.

We treat these three cases in order; it turns out that all the other apeirotopes whose groups are subgroups of [3, 3, 4, 3] fall into families related to one of the three, except for those described at the end of Sect. 7. In reference to the cluster polytope $C$, we obtain the most symmetric picture if we take $o$ as the centre of $C$, rather than as the initial vertex of $P$. For convenience, we label each family by the corresponding diagram $\mathcal{A}_{1}$.

$$
\mathcal{A}_{1}\left(3,3, \frac{3}{2}, 4\right)
$$

The cluster polytope here is $C=\{3,3,4\}$; note also that $C$ coincides with the false cluster polytope $C^{\prime}$ (with a different generator $S_{1}^{\prime}$ instead of $S_{1}$ ). In this case, we have the symmetry $T=\left(R_{2} R_{3}\right)^{2}$ of $Q$; since $\operatorname{dim} T=2$, we see that $\tau=\zeta$ in so far as interchanging the cases $\operatorname{dim} R_{0}=3$ and 1 is concerned (note that $\{3,3,4\}$ and $\{3,3,4\}^{\zeta}$ have the same edge-sets, and so the edge-clusters are the same).

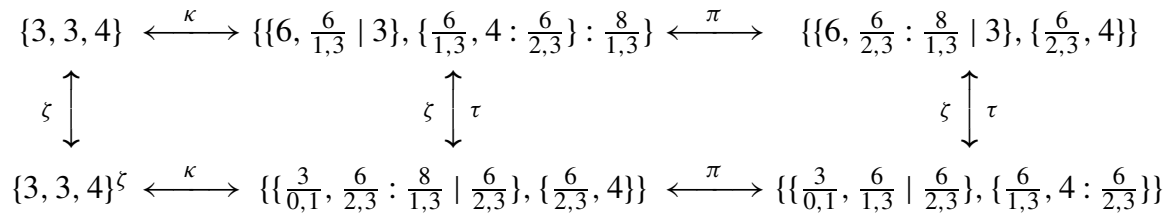

The apeirotope $\left\{\left\{6, \frac{6}{1,3} \mid 3\right\},\left\{\frac{6}{1,3}, 4: \frac{6}{2,3}\right\}\right\}$ at top middle is the facet of the 5apeirotope $\{6,6,4,3\}^{(s)}$ of $[13$, p. 33], and so its facet is the universal $\{6,6 \mid 3\}$. The facet of the apeirotope at bottom middle is (as in Sect. 8) $\{\infty, 6\}_{4,4} \#\{\infty\}$.

The initial edge-cluster is inscribed in a 4 -cube $\{4,3,3\}$, with its vertices at the centres of its 3 -cubical facets. The general edge-clusters are its translates in the tiling $\{4,3,3,4\}$ of $\mathbb{E}^{4}$ by 4 -cubes.

$$
\mathcal{A}_{1}\left(3,4, \frac{3}{2}, 3\right)
$$


The next case is $Q=\left\{\frac{6}{2,3}, 3\right\} \cong\{3,3\} \#\{2\}$. Here, the cluster polytope is $C=$ $\{4,3,3\}$ or $\{4,3,3\}^{\zeta}$, and thus vert $C=\{( \pm 1, \pm 1, \pm 1, \pm 1)\}$. However, comparing Sect. 6 shows that the false cluster polytope is $C^{\prime}=\{3,4,3\}$ or $\{3,4,3\}^{\zeta}$, and so our picture is

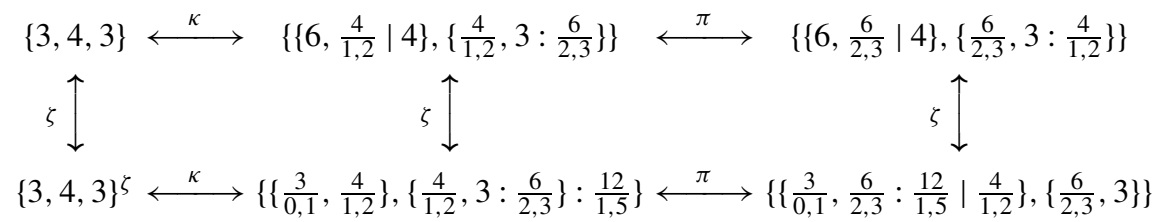

The apeirotope $\left\{\left\{6, \frac{4}{1,2} \mid 4\right\},\left\{\frac{4}{1,2}, 3\right\}\right\}$ at top middle is the facet of the 5-apeirotope $\{6,4,3,3\}^{(s)}$ of $[13$, p. 33], and so its facet is the universal $\{6,4 \mid 4\}$. The facet of the apeirotope at bottom middle is $\{\infty, 4\}_{6,4} \#\{\infty\}$; again, we refer to [19] (see also Remark 4.11).

The initial edge-cluster is inscribed in a 24 -cell $\{3,4,3\}$, with its vertices at 16 of the 24 centres of its facets. The general edge-clusters are its translates in the tiling $\{3,4,3,3\}$ of $\mathbb{E}^{4}$ by 24 -cells.

$$
\mathcal{A}_{1}(2,4,2,3)
$$

This case is the simplest to describe geometrically. Now the vertex-figure is $\left\{\frac{4}{1,2}, 3\right\} \cong\{4,3\} \#\{2\}$, and the cluster polytope is again $C=\{4,3,3\}$, which here coincides with the false cluster polytope $C^{\prime}$.

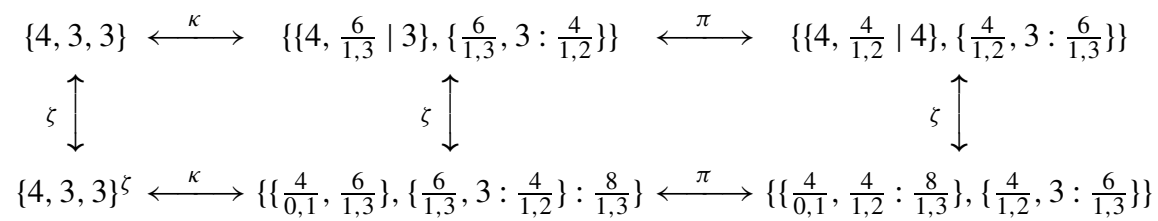

The facets at top middle and right are the universal $\{4,6 \mid 3\}$ and $\{4,4 \mid 4\}$, respectively. The facet of the apeirotope at bottom middle is $\{\infty, 6\}_{6,3} \#\{\infty\}$; once more, we refer to [19] (see also Remark 4.11).

The picture here of the edge-clusters is rather different from that in the other two cases. If $\Lambda$ is the sublattice of $\mathbb{Z}^{4}$ generated by all $( \pm 1, \pm 1, \pm 1, \pm 1)$ (thus $\left.\left[\mathbb{Z}^{4}: \Lambda\right]=8\right)$, then the edge-clusters are centred at $2 \Lambda$, and the vertex-set is $2 \Lambda+$ $(1,1,1,1)$. Thus, in fact, the edge-set of the basic apeirotope $\left\{\left\{4, \frac{4}{1,2} \mid 4\right\},\left\{\frac{4}{1,2}, 3\right.\right.$ : $\left.\left.\frac{6}{1,3}\right\}\right\}$ is the same as that of the cubic tiling $\{4,3,3,4\}$.

Unlike in the other two cases, here this basic apeirotope has a dual $\{\{4,3\}$, $\left.\left\{4, \frac{4}{1,2} \mid 4\right\}\right\}$, and this is the starting point for a very rich family. A partial picture of the relationships among the apeirotopes in this family is illustrated in the following diagram. What is particularly notable is that each of the two tori $\left\{4, \frac{4}{1,2} \mid 4\right\}$ and $\left\{\frac{4}{1,2}, 4: \frac{4}{1,2}\right\}$ occurs as vertex-figure with $\operatorname{dim} R_{0}$ taking each possible value $0,1,2$, 
or 3 (we have not listed the two free abelian apeirotopes).

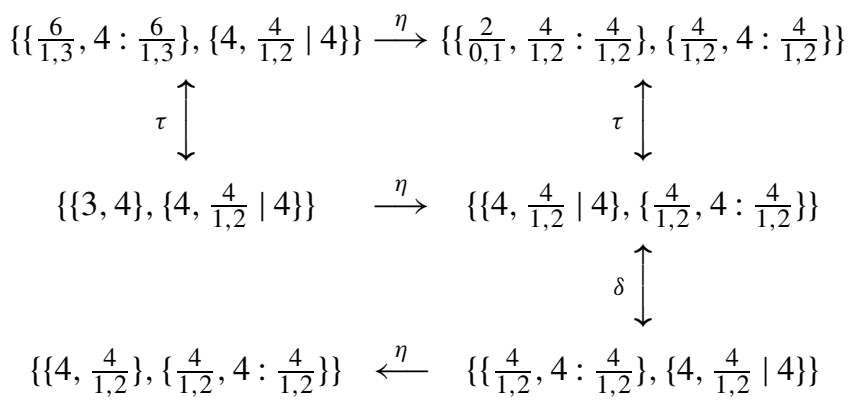

Recall that $\left\{\frac{2}{0,1}\right\}$ is the zigzag apeirogon.

To keep track of the changes of generators implied by the diagram, it is helpful to choose the initial ones as simply as possible (and so we make a change from the previous ones implied by the duality). A particularly suitable choice, which respects the decomposition $\mathbb{E}^{4}=\mathbb{E}^{2} \oplus \mathbb{E}^{2}$ implicit in the tori, is those of the group of $\left\{\{3,4\},\left\{4, \frac{4}{1,2}\right\}\right\}$ :

$$
\begin{aligned}
& R_{0}: x \mapsto\left(1-\xi_{3}, \xi_{2}, 1-\xi_{1}, \xi_{4}\right), \\
& R_{1}: x \mapsto\left(\xi_{2}, \xi_{1}, \xi_{3}, \xi_{4}\right), \\
& R_{2}: x \mapsto\left(\xi_{3}, \xi_{4}, \xi_{1}, \xi_{2}\right), \\
& R_{3}: x \mapsto\left(\xi_{1}, \xi_{2}, \xi_{3},-\xi_{4}\right) .
\end{aligned}
$$

It is easily checked that these do indeed correspond to those of $\mathcal{A}_{1}(2,4,2,3)$ in the reverse order.

Before we move on, we identify some of the facets that occur here and show that some at least of the apeirotopes are universal. Beginning at the top, $\left\{\frac{6}{1,3}, 4: \frac{6}{1,3}\right\} \cong$ $\{6,4\}_{6}$, which is self-Petrie (see [19, Sects. 6 and 7] for more details). In fact, it appears in the form $\{6,4\}_{6} \#\{2\}$; the skew faces of the original are Petrie polygons of 3 -cubes and are lifted in $\mathbb{E}^{4}$ to Petrie polygons of octahedra. Next, still at the top, $\left\{\frac{2}{0,1}, \frac{4}{1,2}: \frac{4}{1,2}\right\}$ stands for the Petrial of the square tessellation $\{4,4\}$, which appears in its own right as $\left\{4, \frac{4}{1,2}\right\}$ near the bottom. The apeirotope $\left\{\{3,4\},\left\{4, \frac{4}{1,2} \mid 4\right\}\right\}$ (which we prove in Theorem 9.2 to be universal) is the facet of the 5-apeirotope denoted $\{3,4,4,3\}^{(s)}$ in the table of $\left[13\right.$, p. 33]. Finally, at bottom left, $\left\{4, \frac{6}{1,3} \mid 4\right\}$ is the universal Petrie-Coxeter apeirohedron $\{4,6 \mid 4\}$, and the apeirotope itself is the facet of the regular 5-apeirotope denoted $\{4,6,3,4\}^{(s)}$ in the same table.

Moreover, since they are derived from diagrams $\mathcal{A}_{1}(2,4,2,3)$ or $\mathcal{A}_{2}(4,4)$, and these can be recovered from the groups of the apeirotopes, we have

Theorem 9.2 The following apeirotopes are universal:

$$
\begin{aligned}
& \left\{\left\{\frac{4}{1,2}, 4: \frac{4}{1,2}\right\},\left\{4, \frac{4}{1,2} \mid 4\right\}\right\} \cong\left\{\{4,4\}_{4},\{4,4 \mid 4\}\right\}, \\
& \left\{\left\{4, \frac{4}{1,2} \mid 4\right\},\left\{\frac{4}{1,2}, 4: \frac{4}{1,2}\right\}\right\} \cong\left\{\{4,4 \mid 4\},\{4,4\}_{4}\right\},
\end{aligned}
$$




$$
\begin{array}{r}
\left\{\{3,4\},\left\{4, \frac{4}{1,2} \mid 4\right\}\right\} \cong\{\{3,4\},\{4,4 \mid 4\}\}, \\
\left\{\left\{4, \frac{4}{1,2} \mid 4\right\},\left\{\frac{4}{1,2}, 3\right\}\right\} \cong\{\{4,4 \mid 4\},\{4,3\}\} .
\end{array}
$$

For the last two, again compare [18].

Of course, we must also consider the effects of $\kappa$ and $\pi$. We note first that the vertex-figure $\left\{\frac{4}{1,2}, 4: \frac{4}{1,2}\right\}$ is preserved by $\kappa$, with a minor change of generators; as the notation indicates, the torus is also self-Petrie, but the isometry that takes the original into its Petrial does not respect the decomposition $\mathbb{E}^{4}=\mathbb{E}^{2} \oplus \mathbb{E}^{2}$. Thus applying $\kappa$ or $\pi$ in these cases cannot change the type of the apeirotope, although it will produce a different congruent copy.

However, under $\kappa$, the vertex-figure $\left\{4, \frac{4}{1,2} \mid 4\right\}$ becomes $\left\{\frac{4}{1,2}, \frac{4}{1,2} \mid \frac{4}{1,2}\right\}$, an isomorphic but not congruent copy, while $\left\{4, \frac{4}{1,2} \mid 4\right\}^{\pi}=\left\{\frac{8}{1,3}, \frac{4}{1,2}: 4,4\right\}$ (these polygons $\left\{\frac{8}{1,3}\right\}$ are Petrie polygons of 4-cubes), and further applying $\kappa$ changes $\left\{\frac{8}{1,3}\right\}$ into a non-congruent polygon of the same kind (and each zigzag $\{4\}$ into $\left\{\frac{4}{1,2}\right\}$ ). Theorem 4.12 tells us how the various data of the corresponding apeirotopes change under $\kappa$. Ignoring the free abelian apeirotope, for the initial effects of $\kappa$, we have here

$$
\begin{aligned}
& \left\{\{3,4\},\left\{4, \frac{4}{1,2} \mid 4\right\}\right\} \stackrel{\kappa}{\longleftrightarrow}\left\{\left\{6, \frac{4}{1,2} \mid 4\right\},\left\{\frac{4}{1,2}, \frac{4}{1,2} \mid \frac{4}{1,2}\right\}\right\},
\end{aligned}
$$

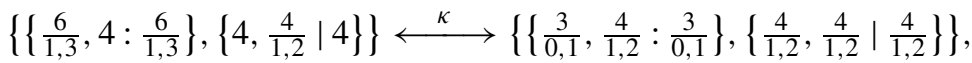



The first facet on the right is isomorphic to the Petrie-Coxeter apeirohedron $\{6,4 \mid 4\}$, and the apeirotope itself is the facet of the 5-apeirotope denoted $\{6,4,4,3\}^{(s)}$ in $[13$, p. 33]; the second is that denoted $\{\infty, 4\}_{., * 3}$ in [19]. We have additionally noted the hole in the third; other than that, we know little about this apeirohedron. We shall not list the effects of $\pi$ (as usual, the data can be found using Theorem 4.9), because we have not identified any facets of interest among the resulting apeirotopes; we merely recall that $\pi$ is not valid for $\left\{\{3,4\},\left\{4, \frac{4}{1,2} \mid 4\right\}\right\}$ but will work on the other five apeirotopes in the diagram.

We conclude the section by seeing how the apeirotopes in case $q=4$ discussed at the end of Sect. 7 fit into the present context. Translated into coordinate terms, the generating reflexions of the vertex-figure $\left\{\frac{8}{1,3}, 4: \frac{8}{1,3}\right\}$ are

$$
\begin{aligned}
& R_{1}: x \mapsto\left(\xi_{3}, \xi_{4}, \xi_{1}, \xi_{2}\right), \\
& R_{2}: x \mapsto\left(\xi_{1},-\xi_{2}, \xi_{4}, \xi_{3}\right), \\
& R_{3}: x \mapsto\left(\xi_{1},-\xi_{2}, \xi_{3},-\xi_{4}\right) .
\end{aligned}
$$

The four reflexions $R_{0}$ take the common form

$$
x \mapsto\left(1-\xi_{1}, \pm \xi_{2}, \pm\left(\xi_{3}, \xi_{4}\right)\right)
$$

with an obvious meaning. The plus sign in the second coordinate gives $\operatorname{dim} R_{0}=1$ or 3 , while that in the third and fourth coordinates (together) gives $\operatorname{dim} R_{0}=2$ or 3 
(and, in case there is any doubt, all minuses gives the case $\operatorname{dim} R_{0}=0$ of the free abelian apeirotope). In view of what we said before, the vertices and edges are those of the cubic tiling $\{4,3,3,4\}$, which also contains the faces $\{4\}$ when $\operatorname{dim} R_{0}=2$ or 3 (the faces in the other two cases are $\left\{\frac{2}{0,1}\right\}=\{\infty\} \#\{2\}$ ); it is abundantly clear that each group is a subgroup of $[4,3,3,4]$.

\section{Concluding Remarks}

In the first paragraph of Sect. 1, it was implicit in what we said that the next item in a dimension-by-dimension classification of regular polytopes and apeirotopes should be that of the four-dimensional apeirohedra. However, what we have done here has thrown up many examples of such apeirohedra whose existence we had hitherto not suspected. It was already clear that this classification problem was not readily soluble; now we know that it will be even less tractable than previously thought.

This suggested aiming at a different target, namely, the case of nearly full rank in the sense of [13, Remark 6.6] (that is, one less than the dimension in the finite case, or the dimension for apeirotopes). The apeirohedra of [19] and the polyhedra of [14] are of nearly full rank, as are the apeirotopes treated in this paper. More recently, in [15] we have classified the polytopes of nearly full rank in higher dimensions; however, though the treatment there relies on the results of this and previous papers, and several of the techniques we use generalize in an expected way, this new material is too extensive to be incorporated here.

Finally, it is appropriate here to make a sort of apology. In our treatment in [14] of the four-dimensional regular polyhedra, we rather casually dismissed the applications of the operations $\kappa$ and $\pi$. As a result, we failed to notice that some of these polyhedra were interesting in their own right. In particular, we have found here faithful realizations of the polyhedron $\{4,6\}_{5}$, its dual $\{6,4\}_{5}$, and their Petrials $\{5,6\}_{4}$ and $\{5,4\}_{6}$.

Acknowledgements We express our grateful thanks to Egon Schulte for his careful reading of a previous draft of this paper, for his extensive and very helpful comments on it, and for a useful telephone conversation. We also wish to thank one of the referees for several suggestions.

\section{References}

1. Arocha, J.L., Bracho, J., Montejano, L.: Regular projective polyhedra with planar faces, I. Aequ. Math. 59, 55-73 (2000)

2. Bracho, J.: Regular projective polyhedra with planar faces, II. Aequ. Math. 59, 160-176 (2000)

3. Coxeter, H.S.M.: Regular skew polyhedra in 3 and 4 dimensions and their topological analogues. Proc. London Math. Soc. (2) 43, 33-62 (1937). Reprinted with amendments in Twelve Geometric Essays, pp. 76-105. Southern Illinois University Press, Carbondale (1968)

4. Coxeter, H.S.M., Moser, W.O.J.: Generators and Relations for Discrete Groups, 4th edn. Springer, Berlin (1980)

5. Dress, A.W.M.: A combinatorial theory of Grünbaum's new regular polyhedra, I: Grünbaum's new regular polyhedra and their automorphism group. Aequ. Math. 23, 252-265 (1981)

6. Dress, A.W.M.: A combinatorial theory of Grünbaum's new regular polyhedra, II: complete enumeration. Aequ. Math. 29, 222-243 (1985) 
7. Frucht, R., Graver, J.E., Watkins, M.E.: The groups of the generalized Petersen graphs. Proc. Camb. Philos. Soc. 70, 211-218 (1971)

8. Grünbaum, B.: Regular polyhedra—old and new. Aequ. Math. 16, 1-20 (1977)

9. McMullen, P.: Realizations of regular polytopes. Aequ. Math. 37, 38-56 (1989)

10. McMullen, P.: The regular polyhedra of type $\{p, 3\}$ with $2 p$ vertices. Geom. Dedic. 43, 285-289 (1992)

11. McMullen, P.: Realizations of regular apeirotopes. Aequ. Math. 47, 223-239 (1994)

12. McMullen, P.: The groups of the regular star-polytopes. Can. J. Math. 50(2), 426-448 (1998)

13. McMullen, P.: Regular polytopes of full rank. Discrete Comput. Geom. 32, 1-35 (2004)

14. McMullen, P.: Four-dimensional regular polyhedra. Discrete Comput. Geom. 38, 355-387 (2007)

15. McMullen, P.: Regular polytopes of nearly full rank (2009, in preparation)

16. McMullen, P., Monson, B.R.: Realizations of regular polytopes, II. Aequ. Math. 65, 102-112 (2003)

17. McMullen, P., Schulte, E.: Constructions for regular polytopes. J. Comb. Theory, Ser. A 53, 1-28 (1990)

18. McMullen, P., Schulte, E.: Regular polytopes from twisted Coxeter groups and unitary reflexion groups. Adv. Math. 82, 35-87 (1990)

19. McMullen, P., Schulte, E.: Regular polytopes in ordinary space. Discrete Comput. Geom. 17, 449-478 (1997)

20. McMullen, P., Schulte, E.: Abstract Regular Polytopes. Encyclopedia of Mathematics and its Applications, vol. 92. Cambridge University Press, Cambridge (2002) 